\title{
Theoretical and Experimental Study of the Excess Thermodynamic Properties of Highly Nonideal Liquid Mixtures of Butanol Isomers + DBE
}

Leon de Villiers Engelbrecht, Riccardo Farris, Tudor Vasiliu, Monica Demurtas, Alessandra Piras, Flaminia Cesare Marincola, Aatto Laaksonen, ${ }^{*}$ Silvia Porcedda, ${ }^{*}$ and Francesca Mocci*

Cite This: J. Phys. Chem. B 2021, 125, 587-600

Read Online

ACCESS | Lلll Metrics \& More | 回 Article Recommendations

Supporting Information

ABSTRACT: Binary alcohol + ether liquid mixtures are of significant importance as potential biofuels or additives for internal combustion engines and attract considerable fundamental interest as model systems containing one strongly $\mathrm{H}$-bonded self-associating component (alcohol) and one that is unable to do so (ether), but that can interact strongly as a H-bond acceptor. In this context, the excess thermodynamic properties of these mixtures, specifically the excess molar enthalpies and volumes $\left(H^{\mathrm{E}}\right.$ and $\left.V^{\mathrm{E}}\right)$, have been extensively measured. Butanol isomer + di- $n$-butyl ether (DBE) mixtures received significant attention because of interesting differences in their $V^{\mathrm{E}}$, changing from negative (1- and isobutanol) to positive (2-and tert-butanol) with increasing alkyl group branching. With the aim of shedding light on the differences in alcohol selfassociation and cross-species H-bonding, considered responsible for the observed differences, we studied representative 1- and 2-butanol $+\mathrm{DBE}$

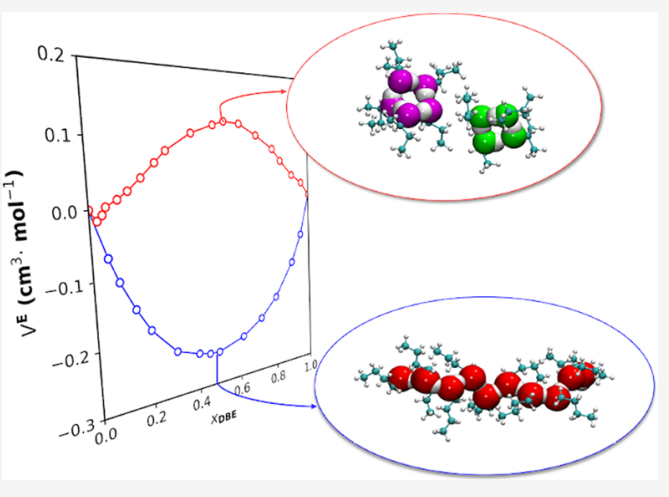
mixtures by molecular dynamics simulations and experimental excess property measurements. The simulations reveal marked differences in the self-association of the two isomers and, while supporting the existing interpretations of the $H^{\mathrm{E}}$ and $V^{\mathrm{E}}$ in a general sense, our results suggest, for the first time, that subtle changes in H-bonded topologies may contribute significantly to the anomalous volumetric properties of these mixtures.

\section{INTRODUCTION}

The physical properties of binary liquid mixtures of alcohols with either alkanes or ethers have been the subject of sustained research interest over the past few decades due to both their practical importance as fuel additives in the internal combustion engines and fundamental interest (a mixture of self-associating and non-self-associating components). ${ }^{1-7}$

In fuel applications, oxygenated compounds, e.g., alcohols and ethers, are used and investigated as gasoline/fuel additives with the aim of reducing pollutants from vehicle exhaust emissions by altering combustion characteristics. ${ }^{7-9}$ Higher alcohols, and butanol isomers in particular, are increasingly studied for applications as biofuels since they may offer advantages over the traditional biofuel, ethanol, including better compatibility with the existing engine designs at $85 \%$ per volume compared with the corresponding ethanol-based fuel (E85), and higher energy content per unit volume. ${ }^{8,10}$ Indeed, butanol isomers are included in the international regulation on the promotion of the use of energy from renewable sources. ${ }^{11}$ The related di- $n$ butyl ether (DBE) has been investigated as a blending agent in gasoline. ${ }^{9}$ In the context of fuel applications, there is considerable interest in understanding the physical properties of such alcohol + ether binary mixtures, e.g., excess thermodynamic properties. ${ }^{5,12}$
Measurements of the molar enthalpies of mixing, $\Delta_{\text {mix }} H$, a property coinciding with the excess molar enthalpy, $H^{\mathrm{E}}$, and the excess molar volumes, $V^{\mathrm{E}}$, of binary mixtures of selected butanol isomers and DBE have been reported by several groups over the past 3 decades. ${ }^{2-4,6}$ Both excess thermodynamic properties have been found to increase in magnitude in the order 1-butanol ( $n$ butanol) $<2$-methyl-1-propanol (isobutanol) $<2$-butanol (secbutanol) $<2$-methyl-2-propanol (tert-butanol; tert-butyl alcohol), with the $V^{\mathrm{E}}$ values showing an interesting change in sign from negative at all compositions for 1-butanol and isobutanol, to predominantly positive for 2-butanol and positive at all compositions for tert-butyl alcohol + DBE mixtures. These excess property trends have been interpreted as due to the differences in the balance of intermolecular interaction contributions arising from the breakage of alcohol-alcohol hydrogen bonds (H-bonds) upon mixing, the formation of

Received: November 8, 2020

Revised: December 16, 2020

Published: January 11, 2021 
alcohol-DBE H-bonds within the mixtures, and other nonspecific intermolecular interactions. For example, the observed $V^{\mathrm{E}}$ trend of these mixtures has been interpreted as largely due to steric effects on alcohol-DBE cross-species H-bonding, where the alkyl chain branching in the vicinity of the hydroxyl groups of 2- and tert-butanol hinders $\mathrm{H}$-bonding with DBE, resulting in a smaller negative $V^{\mathrm{E}}$ contribution from this interaction type compared to 1 - and isobutanol.,

Kammerer et al. $^{4}$ applied the extended real association solution (ERAS) model to 1-butanol and 2-butanol + DBE mixtures with the aim to obtain the simultaneous description of $H^{\mathrm{E}}$ and $V^{\mathrm{E}}$ expressed by a set of system-specific parameters. As both the mixtures exhibit strong hydrogen bonding, the chemical contribution was assumed to be dominant, with respect to (w.r.t.) the physical contribution, in the calculation of each excess properties under examination, and a fixed value for the physical parameter was used. A good representation of the experimental data was obtained using the parameters previously obtained by a least-squares treatment. For 2-butanol + DBE, the model calculations result in a much lower value for the association constant between alcohol and ether, $K_{\mathrm{AB}}$, than for the system with 1-butanol.

Numerous experimental studies of alcohol + alkane mixtures by, e.g., ultrasonic measurements, vibrational or NMR spectroscopic techniques, and neutron scattering, have revealed varying degrees of alcohol self-association via $\mathrm{H}$-bonding. ${ }^{1,13,14}$ Moreover, computational molecular modeling methods, notably Monte Carlo (MC) and molecular dynamics (MD) simulations, have provided additional detailed molecular-level information on the nature of alcohol $\mathrm{H}$-bonding and self-association in such mixtures, e.g., H-bonded cluster size distributions. ${ }^{15-20}$ Conversely, alcohol + ether binary liquid mixtures have received considerably less computational consideration; ${ }^{21}$ in fact, to the best of our knowledge, no computer simulation studies have been reported for any butanol isomer + DBE mixtures described above. To address this gap, we combine here the measurement of the thermodynamic properties for two such mixture series, 1butanol and 2-butanol + DBE (molecular structures shown in Figure 1), with $\mathrm{MD}$ computer simulations performed over the

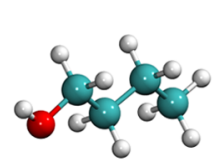

(a)

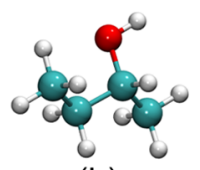

(b)

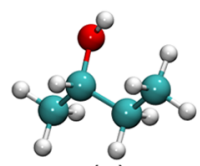

(c)

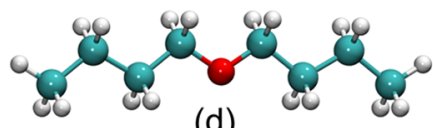

(d)

Figure 1. Molecular structures of (a) 1-butanol, (b, c) 2-butanol ( $R$ and $S$ stereoisomers, respectively), and (d) di- $n$-butyl ether (DBE). Hydrogen atoms are shown in white, carbon in cyan, and oxygen in red.

entire mixture composition ranges, specifically with the aim of studying H-bonded alcohol self-association and its dependence on the hydroxyl position in a linear hydrocarbon skeleton, how differences in the association related to the existing explanations of their much-studied excess thermodynamic properties, ${ }^{2-4,6}$ and how the dramatic differences of such properties among the two alcohol mixtures are related to the different molecular organization.

\section{METHODS}

2.1. Sample Preparation. The substances employed in this study were purchased from Sigma, and their relevant physical properties are reported in Table 1 . Before using, all solvents were

Table 1. Chemical Abstract Serial Number (CASNR), Molar Masses, $M$, Purities Expressed as Mass Fraction, $w_{\mathrm{J}}$, and Experimental and Literature ${ }^{22}$ densities, $\rho$, at $298.15 \mathrm{~K}$ of Chemicals Used in the Experiments

$\begin{array}{llcccc}\text { compound } & \text { CASNR } & \begin{array}{c}M^{a} \\ \left(\mathrm{~g} \cdot \mathrm{mol}^{-1}\right)\end{array} & w_{\mathrm{J}} & \begin{array}{c}\rho_{\text {exp }} \\ (\mathrm{kg} \cdot \mathrm{m}\end{array} & \begin{array}{c}\rho_{\text {lit }} \\ \left(\mathrm{kg} \cdot \mathrm{m}^{-3}\right)\end{array} \\ \text { DBE } & 142-96-1 & 130.23 & 0.993 & 764.17 & 764.10 \\ \text { 1-butanol } & 71-36-3 & 74.12 & 0.994 & 805.97 & 805.75 \\ \text { 2-butanol } & 78-92-2 & 74.12 & 0.995 & 802.19 & 802.41\end{array}$

${ }^{a}$ Calculated from the atomic weight of elements recommended by IUPAC. ${ }^{23}$

degassed for about $2 \mathrm{~h}$ by means of an ultrasonic device (WVR model USC100T, $45 \mathrm{kHz}, 30 \mathrm{~W}$ ). Water was obtained by distillation and used immediately after preparation.

2.2. Calorimetry. Heats of mixing were determined by means of a flow microcalorimeter (model 2277, LKB-producer $\mathrm{AB}$, Bromma, Sweden). Fully automatic burets (ABU80, Radiometer, Copenhagen, Denmark) were used to pump the liquid into the LKB unit. The molar flow rate $\Phi_{i}$, of component $i$ flowing into the mixing cell, is given by

$$
\Phi_{i}=\Psi_{i} \frac{\rho_{i}}{M_{i}}
$$

where $\Psi_{i}$ is the volumetric flow rate, $\rho_{i}$ is the density, and $M_{i}$ is the molar mass. The molar excess enthalpies have been evaluated from the formula

$$
H^{\mathrm{E}}\left(x_{1}\right)=\frac{P\left(x_{1}\right)}{\Phi_{\mathrm{T}}}
$$

where $P\left(x_{1}\right)$ is the actual value of the power associated with the formation of a given mixture, obtained by comparison of the raw experimental value with the one corresponding to an electrical calibration experiment and $\Phi_{\mathrm{T}}$ is the molar flow rate of the mixture. All enthalpy measurements were carried out at 298.15 $\mathrm{K}$ and at $0.1 \mathrm{MPa}$. The accuracy of the LKB bath temperature is $0.01 \mathrm{~K}$. The reliability of the apparatus and procedure adopted were checked periodically by performing $H^{\mathrm{E}}$ measurements on the test system benzene + cyclohexane. Our results concerning this system differed by $<2 \%$ from reliable literature data ${ }^{24}$ over the entire composition range. The whole set of $H^{\mathrm{E}}$ experimental data is reported in Table S1.

2.3. Volumetry. The necessary density data for the determination of the molar flow in the calorimetric determinations and of the excess molar volumes were measured using a vibrating tube densitometer (model DMA 58, Anton Paar, Graz, Austria) with a reproducibility of $1 \times 10^{-2} \mathrm{~kg} \mathrm{~m}^{-3}$. Accuracy in the temperature was better than $\pm 1 \times 10^{-2} \mathrm{~K}$ by means of an internal temperature control device that applies the Peltier principle to maintain isothermal conditions. Densitometer calibration was performed before every run using a double reference (water and dry air).

The liquid mixtures were prepared by weighing in septumcapped vials of approximately $8 \mathrm{~mL}$ using needles and syringes to transfer liquids. 
The molar excess volumes $V^{\mathrm{E}}$ were calculated from the following equation

$$
V^{\mathrm{E}}=\frac{x_{1} M_{1}+x_{2} M_{2}}{\rho}-\frac{x_{1} M_{1}}{\rho_{1}}-\frac{x_{2} M_{2}}{\rho_{2}}
$$

where $x_{1}$ and $x_{2}$ are the mole fractions, $M_{1}$ and $M_{2}$ are the molar masses, $\rho_{1}$ and $\rho_{2}$ are the densities of the two pure components, and $\rho$ is the density of the mixture. The procedure was checked by comparison of our experimental $V^{\mathrm{E}}$ data of the water + ethanol system with reliable literature data. ${ }^{25}$ Our experimental data differed by less than $0.8 \%$ in the whole composition range. The whole set of $V^{\mathrm{E}}$ experimental data is reported in Table S2.

2.4. Computational Details. Molecular dynamics (MD) computer simulations were performed with the AMBER 18 package $^{26}$ using both central processing unit (CPU) and graphics processing unit (GPU) versions of PMEMD. ${ }^{27} 1$ and 2-butanol (racemic mixture of $R$ and $S$ stereoisomers) and DBE were modeled with the general AMBER force field $(\mathrm{GAFF})^{28}$ using Antechamber software to generate the library files. Atomic partial charges were calculated according to the GAFF protocol at the Hartree-Fock (HF) theory level with the basis set $6-31 \mathrm{G}^{*}$. Electronic structure calculations were performed using Gaussian 09. ${ }^{29}$ MD simulations were carried out at a temperature of $298.15 \mathrm{~K}$ and $0.1 \mathrm{MPa}$ pressure, which were regulated using the Berendsen weak coupling thermostat/ barostat $^{30}$ using coupling times of 0.1 and $0.5 \mathrm{ps}$, respectively. Periodical removal (every $2 \mathrm{ps}$ ) of the center of mass velocity was done to minimize the "flying ice cube" phenomenon. ${ }^{31} \mathrm{~A}$ nonbonded interaction cut-off was set at $12 \AA$, with long-range electrostatic interactions treated using the particle-mesh Ewald (PME) algorithm. Appropriate values of isothermal compressibility, $\kappa_{\mathrm{T}}$, of the various mixtures under these conditions were obtained by interpolation of the data reported by Montero and co-workers. $^{7-10}$

2.4.1. Starting Configurations. Each simulated system contained a total of 400 molecules in a cubic cell with the mole fraction $\mathrm{DBE}, x_{\mathrm{DBE}}$, covering the entire composition range. Specifically, in addition to the simulations of the three pure liquids, for each alcohol, a total of eight simulations of DBE mixtures were performed with the following $x_{\mathrm{DBE}}=\{0.12,0.25$, $0.375,0.50,0.625,0.75,0.88,0.94\}$. The molecules were packed in a simulation cubic box, with periodic boundaries, of side length between 50 and $54 \AA$, depending on the system, using the PACKMOL program. ${ }^{32}$

2.4.2. Equilibration Protocol and Production Details. Following an initial steepest-descent energy minimization (10 000 steps), each system was equilibrated first under constant temperature and volume conditions (NVT, $1 \mathrm{~ns})$, and then under constant pressure (NPT, $1 \mathrm{~ns})$; during this time period, the temperature, pressure, and density reached the equilibrium. Production simulations of at least $50 \mathrm{~ns}$ were similarly performed under NPT conditions. All simulations were carried out using a time step of 2 fs with SHAKE constraints ${ }^{33}$ on hydrogen atoms (default tolerance $=0.00001)$.

Statistical errors in the calculated thermodynamic properties were evaluated using the block averaging method. ${ }^{34}$ More detailed information on this procedure may be found in the Supporting Information.

For the H-bonded alcohol cluster analysis, we developed a script, described in the Supporting Information, written in tool command language $(\mathrm{tcl})$, that makes use of $\mathrm{VMD}^{35}$ and its atom selection capabilities. All of the pictures of the molecular configurations were produced using VMD.

\section{RESULTS}

The measured enthalpies of mixing, $H^{\mathrm{E}}$, and excess molar volumes, $V^{\mathrm{E}}$, of 1- and 2-butanol + DBE mixtures at $298.15 \mathrm{~K}$, $0.1 \mathrm{MPa}$, are compared with those computed from classical MD simulations in Figures 2 and 3; corresponding tabulated data,

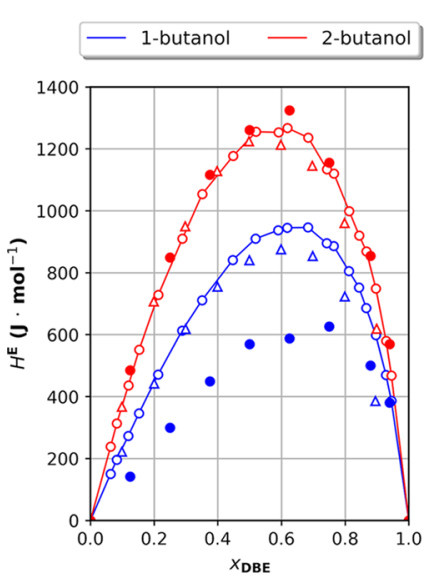

Figure 2. Enthalpies of mixing $\left(H^{\mathrm{E}}\right)$ of 1- (blue data markers) and 2butanol (red markers) + DBE binary mixtures, with the composition expressed as a function of $x_{\mathrm{DBE}}$, temperature $298.15 \mathrm{~K}, 0.1 \mathrm{MPa}$. Open circles connected by the solid line: present experimental measurements; open triangles: measurements of Kammerer and Lichtenthaler; ${ }^{4}$ solid (filled) circles: MD computer simulation results (full details in the main text). Error bars for the simulation data, see Tables S4 and S5, are smaller than the data marker size and have been omitted for clarity. Experimental uncertainties are given in Table S1.

both experimental and computational, are reported in Tables S1-S5 in the Supporting Information. Also shown in Figures 2 and 3, for comparison, are experimental results from selected literature sources, ${ }^{4,6,36}$ which may be seen to be in very good agreement with our measurements; the results from other earlier reports $^{2,3}$ are also in good agreement but are not shown here in

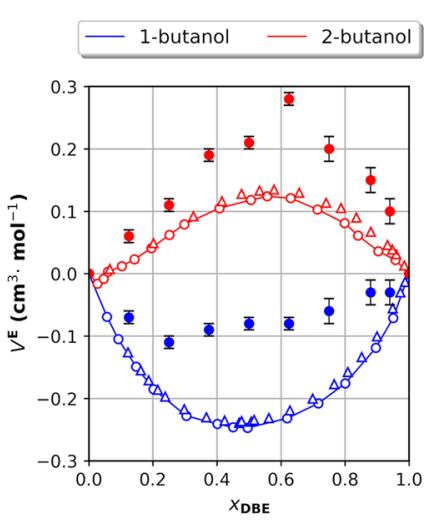

Figure 3. Excess molar volumes $\left(V^{ \pm}\right)$of 1- (blue markers) and 2butanol (red) + DBE mixtures with the composition expressed as a function of $x_{\mathrm{DBE}}$ at $298.15 \mathrm{~K}, 0.1 \mathrm{MPa}$. As in Figure 2, open circle markers (joined by solid lines) show the present experimental measurements, open triangles show the previous measurements of Bernazzani et al. ${ }^{6,36}$ for comparison, and filled circles show the MD results. Error bars on the simulation data represent 1 standard deviation of the mean. Experimental uncertainties are given in Table S2. 

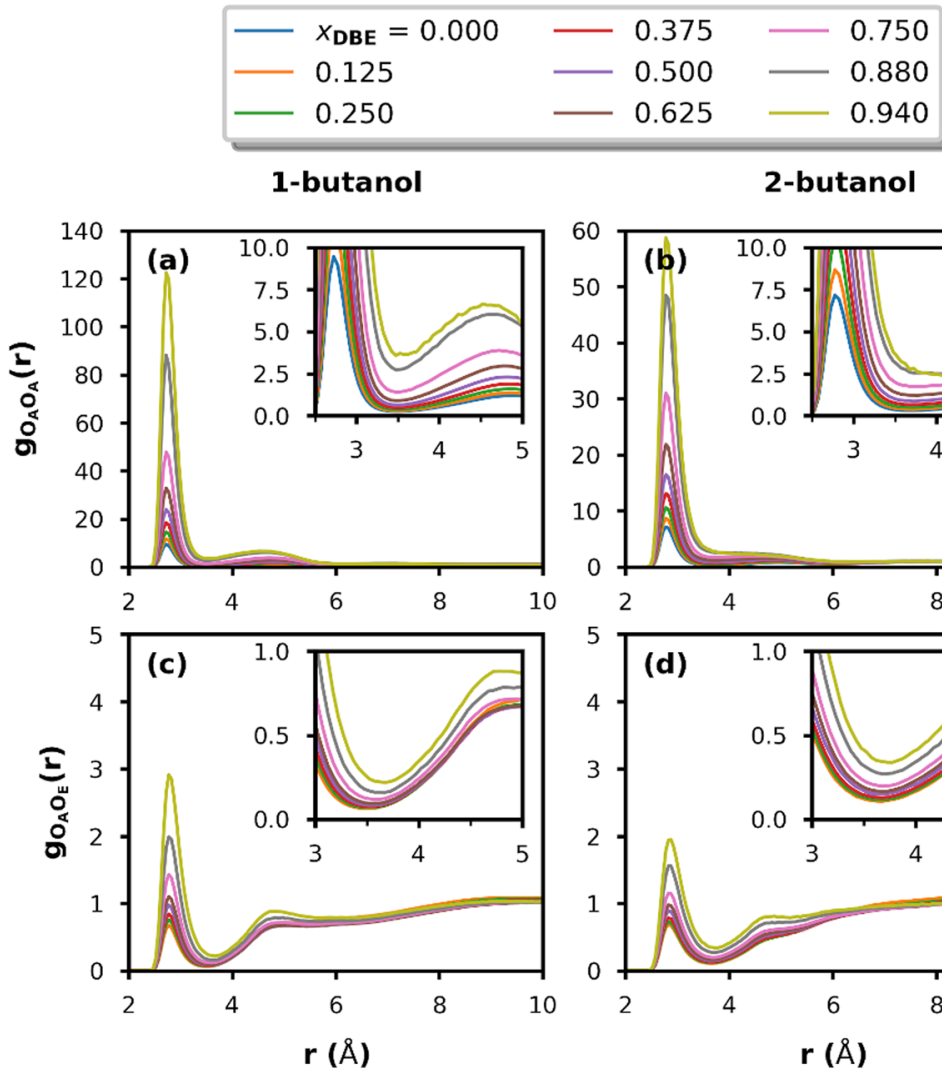

-butanol
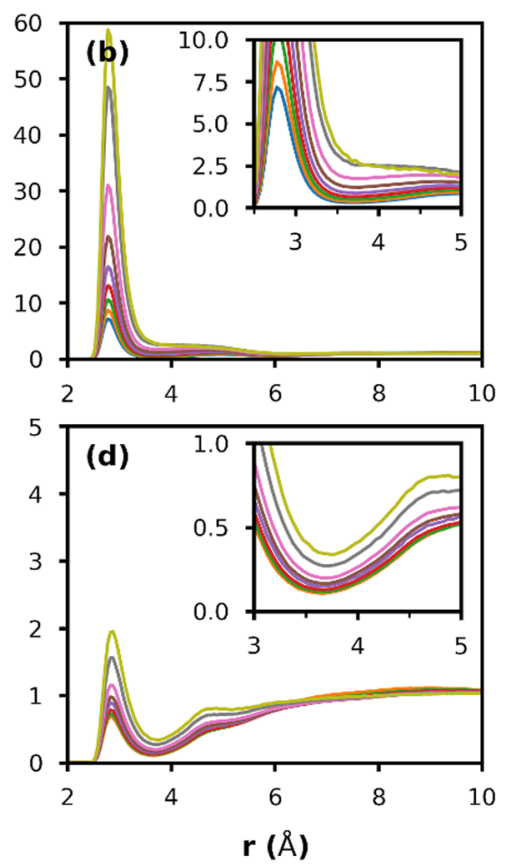

Figure 4. Radial distribution functions, $g_{\mathrm{OO}}(r)$, computed for oxygen atoms from simulated 1- and 2-butanol + DBE mixtures. Top: alcohol-alcohol oxygen atom $\left(\mathrm{O}_{\mathrm{A}}-\mathrm{O}_{\mathrm{A}}\right)$ pair in (a) 1-butanol and (b) 2-butanol and their DBE mixtures. Bottom: alcohol-DBE $\left(\mathrm{O}_{\mathrm{A}}-\mathrm{O}_{\mathrm{E}}\right)$ oxygen pair in (c) 1- and (d) 2-butanol + DBE mixtures. Insets show expansions of the respective first RDF minimum regions, which were used as the cut-off distance for the evaluation of $\mathrm{H}$-bonded neighbors.

the interest of clarity. Considering first the $H^{\mathrm{E}}$ plots, the simulation results indicate a much larger $H^{\mathrm{E}}$ for 2-butanol mixing than for 1-butanol, in full agreement with the experimental data. The computed $H^{\mathrm{E}}$ for 2-butanol mixtures is very close to the experimental values, while for 1-butanol mixtures they have a trend similar to the experimental values showing a similar asymmetry with both maxima around $x_{\mathrm{DBE}}=$ 0.7 , but are generally lower than experiment. It should be noted at this stage that the magnitude of deviations from experiment observed for the simulated 1-butanol + DBE mixtures is not uncommon among computer simulation studies using standard classical force fields, e.g., optimized potentials for liquid simulations-all atoms (OPLS-AA), ${ }^{37}$ often reporting larger deviations, ${ }^{38}$ and will be further discussed below. On the other hand, the agreement between simulations and experiments obtained for 2-butanol mixtures is remarkable.

The overall experimental $V^{ \pm}$trends in Figure 3 are well reproduced by the $\mathrm{MD}$ simulations, and, very importantly, the signs of the data correspond to the experiment, with DBE mixtures with 2-butanol showing a positive excess volume while with 1-butanol a negative one. The simulated $V^{\mathrm{E}}$ are higher than the experimental ones for both isomers and at all compositions, with the 1-butanol mixture data again showing larger discrepancies and greater asymmetry w.r.t. compositions. Such discrepancies are, however, not surprising, considering related computer simulation studies of binary liquid mixtures, since the reproduction of small experimental $V^{\mathrm{E}}$ values is evidently challenging when using standard classical force fields, often resulting in qualitative differences; the reader is referred to the simulation study of S-shaped excess thermodynamic properties by Miroshnichenko and Vrabec, ${ }^{39}$ and references therein. Deviations between the experimental and simulated excess properties for binary liquid mixtures are often ascribed to limitations of classical force field combination (or combining) rules for describing interactions among unlike molecules, and it has been demonstrated that this agreement may be improved by the application of certain scaling procedures to the computation of intermolecular interactions. ${ }^{39}$ Overall, the currently reported simulation excess thermodynamic results reproduce the main features of the experimental observations, and, in view of this validation, can be considered a realistic model for a deeper understanding of the origin of the properties of interest.

\section{DISCUSSION}

4.1. Enthalpies of Mixing and Structural Organization. The $H^{\mathrm{E}}$ measurements of butanol isomer + DBE binary liquid mixtures have generally been interpreted as resulting from a balance of two energetic contributions of opposite sign: ${ }^{2,4}$

- a positive (endothermic) contribution from the breakage of alcohol-alcohol H-bonds, which characterize the respective pure butanol isomer liquids, upon mixing, and

- a negative (exothermic) contribution from the formation of alcohol-ether H-bonds in the mixture.

For a given alcohol + ether binary mixture, the H-bond enthalpy of two self-associated alcohol molecules is greater than that of an alcohol-ether H-bond, ${ }^{2,40}$ from which it follows that the measured $H^{\mathrm{E}}$ do not necessarily reflect (in the absence of more detailed energetic information) the extent to which 
alcohol-alcohol H-bonds are replaced by alcohol-ether $\mathrm{H}$ bonds upon mixing. Based on this interpretation, the positive $H^{\mathrm{E}}$ values measured for butanol-DBE mixtures have been taken to indicate that the energetic cost associated with breaking of the particular H-bonded structure of the pure alcohol upon mixing with DBE is not fully compensated by the formation of new alcohol-DBE H-bonds. Kammerer and Lichtenthaler ascribed the greater $H^{\mathrm{E}}$ of 2-butanol compared to 1-butanol $+\mathrm{DBE}$ binary mixtures (Figure 2) to a more pronounced formation of 1-butanol-DBE H-bonds, whereas the formation of such crossspecies $\mathrm{H}$-bonds is more sterically hindered for the corresponding 2-butanol mixtures. ${ }^{4}$ Interestingly, Lafuente and co-workers, ${ }^{41,42}$ in their study of excess thermodynamic properties of binary mixtures of 1- and 2-butanol with the cyclic ether tetrahydrofuran (THF) and 2-methyl-THF, proposed the larger $H^{\mathrm{E}}$ values of the 2-butanol mixtures to be predominantly due to a greater degree of breakage of the pure alcohol H-bond associated structure upon mixing, i.e., contribution 1 described above.

While it is generally accepted that pure liquid 1-butanol is more strongly and extensively associated by $\mathrm{H}$-bonding compared to the other butanol isomers, including 2-butanol, ${ }^{43}$ and that it is similarly more strongly self-associated in mixtures with alkanes ${ }^{44}$ and possibly ethers, ${ }^{41}$ it appears that the differences in the $\mathrm{H}$-bonded structures of pure 1- and 2-butanol, the $\mathrm{H}$-bond topologies in mixtures with DBE, or the precise role of these differences in determining $H^{\mathrm{E}}$ of such mixtures have not been studied.

In this context, $\mathrm{MD}$ simulations can provide detailed information on the atomistic-level structure and dynamics of molecular liquids, their mixtures, ${ }^{45-49}$ their solvating properties, ${ }^{50,51}$ and the microstructural and dynamical heterogeneity of mixture of solvents of various degrees of complexity. ${ }^{52-54}$ As discussed in the computer simulation Section 3, our MD computer simulations satisfactorily reproduce the $H^{\mathrm{E}}$ trends of 1- and 2-butanol + DBE mixtures, and thus, we can proceed to investigate the structures of the simulated liquids and liquid mixtures with the aim of understanding the molecular origin of the observed difference in the $H^{\mathrm{E}}$.

Structural studies of $\mathrm{H}$-bonded liquids routinely make use of oxygen-oxygen radial pair distribution functions (RDFs), $g_{\mathrm{OO}}(r)$, computed from the simulation trajectories, which describe the radially averaged distribution of oxygen atoms in the vicinity of any given (average) oxygen atom. This function has a strong experimental connection in the form of X-ray and neutron scattering patterns, ${ }^{55}$ with its typical intense first maximum (typically in the distance range of 2.5-3.0 $\mathrm{A}$ ) taken to indicate the presence of directly $\mathrm{H}$-bonded neighbors; the function may be suitably integrated (typically up to the first minimum) to estimate the average number of such neighbors, also often referred to as the coordination number.

The alcohol oxygen (hereafter abbreviated $\mathrm{O}_{\mathrm{A}}$ ) $\mathrm{RDF}$ around a given $\mathrm{O}_{\mathrm{A}}$ for the simulated 1- and 2-butanol pure liquids and all DBE mixtures studied are shown in Figure 4a,b, respectively. At all mixture compositions studied, the first maxima (i.e., occurring at shortest distances) of the $\mathrm{O}_{\mathrm{A}}-\mathrm{O}_{\mathrm{A}} \mathrm{RDF}$ of simulated 1-butanol and its DBE mixtures are more intense than those of the corresponding 2-butanol systems at the same DBE molar fraction, indicative of a higher degree of alcohol selfassociation via $\mathrm{H}$-bonding. Furthermore, the insets in Figure $4 \mathrm{a}, \mathrm{b}$ show that these first maxima consistently occur at slightly shorter distances for 1-butanol and its mixtures, suggesting a stronger and more compact alcohol-alcohol H-bonding compared to 2-butanol systems; pertinent numerical data may be found in Table S6. For the pure alcohols, these findings are essentially consistent with the $\mathrm{O}_{\mathrm{A}}-\mathrm{O}_{\mathrm{A}}$ RDFs presented by Mesele et al. ${ }^{56}$ in their MD simulation study (temperature 298 $\mathrm{K}$, using the OPLS-AA force field ${ }^{37}$ ) of reorientational motions in pure liquid butanol isomers, though a slightly lower 1-butanol first maximum was reported by these authors (see below).

Considering the $\mathrm{O}_{A}-\mathrm{O}_{\mathrm{A}} \mathrm{RDF}$ s of the simulated 1- and 2butanol + DBE mixtures, the first maxima intensities may be seen to increase with the increasing DBE content, $x_{\mathrm{DBE}}$. It should be stressed at this stage that since the computed RDFs are normalized to the bulk densities of the particles considered (in this case, $\mathrm{O}_{\mathrm{A}}$ ), which decrease with the increasing $x_{\mathrm{DBE}}$, the observed RDF intensity increase does not necessarily correspond to an increase in the number of $\mathrm{H}$-bonded neighbors. Indeed, the $\mathrm{O}_{\mathrm{A}}-\mathrm{O}_{\mathrm{A}}$ coordination numbers, obtained by the integration of these first RDF peaks (to their respective first minima, $R_{\mathrm{OO}}$; see Table $S 6$ ) and plotted in Figure 5, show the

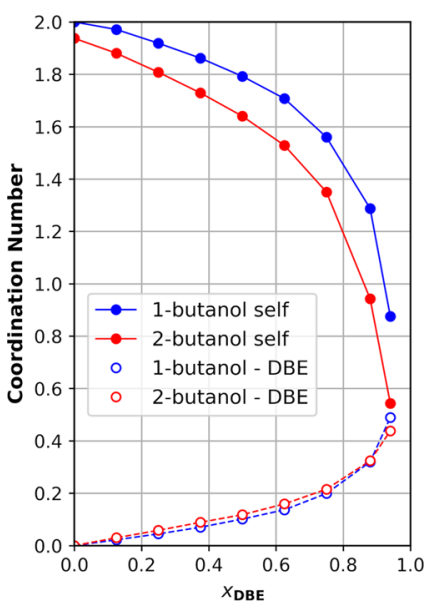

Figure 5. $\mathrm{O}-\mathrm{O}$ coordination numbers, obtained by $\mathrm{RDF}$ integration, plotted as a function of mixture composition, $x_{\mathrm{DBE}}$. Data for 1-butanol (blue) and 2-butanol (red); $\mathrm{O}_{\mathrm{A}}-\mathrm{O}_{\mathrm{A}}$ (alcohol-alcohol) coordination numbers plotted in the solid line, solid circle data markers, and $\mathrm{O}_{\mathrm{A}}-\mathrm{O}_{\mathrm{E}}$ (alcohol-ether) in dashed line, open circle markers.

expected decrease with the increasing $x_{\mathrm{DBE}}$ for both mixture series, i.e., the average number of $\mathrm{H}$-bonded alcohol neighbors of a given alcohol molecule decreases as the alcohol is increasingly diluted with $\mathrm{DBE}$. The $\mathrm{O}_{\mathrm{A}}-\mathrm{O}_{\mathrm{A}}$ coordination numbers for the simulated 1-butanol mixtures are greater than those of the corresponding 2-butanol mixtures at all compositions studied, indicating a higher average number of such $\mathrm{H}$-bonded alcohol-alcohol neighbors, as previously inferred from the $\mathrm{O}_{A}-\mathrm{O}_{\mathrm{A}} \mathrm{RDF}$ intensity differences.

Similar O-O RDFs may be computed to study the crossspecies $\mathrm{H}$-bonding between alcohol and ether (with the oxygen atom, $\mathrm{O}_{\mathrm{E}}$ ) molecules. The $\mathrm{O}_{\mathrm{A}}-\mathrm{O}_{\mathrm{E}} \mathrm{RDF}$ and the corresponding coordination numbers are shown in Figures $4 \mathrm{c}, \mathrm{d}$ and 5, respectively. The $\mathrm{O}_{A}-\mathrm{O}_{E} \mathrm{RDF}$ s exhibit shapes and variations apparently similar to their $\mathrm{O}_{A}-\mathrm{O}_{A}$ counterparts, with those of 1butanol systems consistently more intense, yet the coordination numbers in Figure 4 reveal that the average numbers of $\mathrm{O}_{\mathrm{E}} \mathrm{H}$ bonded to a given $\mathrm{O}_{\mathrm{A}}$ are significantly lower than the corresponding $\mathrm{O}_{\mathrm{A}}-\mathrm{O}_{\mathrm{A}}$ numbers and rather similar for the 1and 2-butanol mixtures studied. While the first of these findings is not unexpected (since a given alcohol molecule can form only a single $\mathrm{H}$-bond with a neighboring $\mathrm{DBE}$ molecule), the 
similarity of the $\mathrm{O}_{A}-\mathrm{O}_{\mathrm{E}}$ coordination numbers for the two alcohols is interesting in view of the fact that previous investigations have attributed the different $H^{\mathrm{E}}$ of these mixtures to more pronounced alcohol-DBE H-bonding for 1-butanol. ${ }^{4}$ Considering the consistently higher 1-butanol $\mathrm{O}_{A}-\mathrm{O}_{\mathrm{A}}$ coordination numbers and consequently lower availability of 1-butanol molecules for $\mathrm{H}$-bonding with DBE, it may indeed be concluded that a larger fraction of the available 1-butanol molecules forms H-bonds with DBE.

The analysis of $\mathrm{O}-\mathrm{O}$ RDFs, as described above, has been extensively used to study $\mathrm{H}$-bonding in computer simulations; however, the additional H-bond criteria have been proposed (both geometric and energetic); the following set of three geometric $\mathrm{H}$-bond criteria are widely cited. ${ }^{57,58}$

1. The distance between the H-bond donor and acceptor oxygen atoms, $\mathrm{O}_{\text {Don }}$ and $\mathrm{O}_{\text {Acc }}$ should be smaller than a certain value, typically chosen as the corresponding $\mathrm{O}_{\text {Don }}-\mathrm{O}_{\text {Acc }} \mathrm{RDF}$ first minimum, hereafter $R_{\mathrm{OO}}$.

2. The distance $R_{\mathrm{OH}}$ between the donor $\mathrm{H}$ and acceptor $\mathrm{O}$ atoms should be smaller than a certain limit, similarly taken as the $\mathrm{O}_{\text {Acc }}-\mathrm{H}_{\text {Don }} \mathrm{RDF}$ first minimum.

3. An angle criterion defining the relative orientations of the donor and acceptor groups; in this study, we define the $\mathrm{H}$ bond $\mathrm{O}_{\text {Don }}-\mathrm{H}_{\text {Don }} \cdots \mathrm{O}_{\text {Acc }}$ obtuse angle $\geq 135^{\circ}$.

In practical $\mathrm{H}$-bond definitions, at least one of the above distance criteria (typically criterion 1 ) is combined with an angle criterion, allowing for the development of algorithms that provide detailed information on simulated $\mathrm{H}$-bond topologies, e.g., fractions of molecules involved in different numbers of $\mathrm{H}$ bonds, the distribution of $\mathrm{H}$-bonded self-associate (or "cluster") sizes, etc. ${ }^{59}$ These $\mathrm{H}$-bond criteria are also incorporated into freely available software packages, e.g., the "hbond" command in the CPPTRAJ program ${ }^{60}$ (a combination of criteria 1 and 3 ), which was used in the present study. The computed average numbers of alcohol-alcohol and alcohol-DBE H-bonds for all simulated 1- and 2-butanol + DBE mixtures are plotted in Figure 6.

Comparison of these $\mathrm{H}$-bond analysis results with those obtained by RDF integration (Figure 5) shows strong similarities w.r.t. the trends and differences between the two alcohols. As regards the actual values, the average number of $\mathrm{H}$ bonds per alcohol molecule obtained by the H-bond analysis procedure is consistently lower than those from $\mathrm{RDF}$ integration

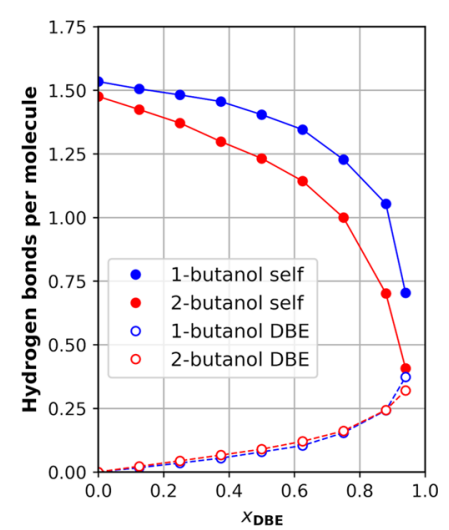

Figure 6. Average number of $\mathrm{H}$-bonds for a given 1- or 2-butanol molecule with other alcohol molecules of the same type (solid circle data markers, solid line) and with DBE (open circle, dashed line). Color code: blue, 1-butanol and red, 2-butanol. for both mixture series; this difference is due to the application of the additional angle criterion. We note that this lower average number of $\mathrm{H}$-bonds per molecule in the simulated pure 1butanol obtained using the hbond tool (1.53) agrees favorably with that reported by Cerar et al. ${ }^{61}$ (1.56) for the AMBER03 force field, as implemented in the GROMACS 5.1 simulation package $^{62}$ (however, using a different set of H-bond criteria). The H-bond analysis described above can provide a wealth of additional information, e.g., the average H-bond distances, angles, and their time evolution, some of which is presented in the Supporting Information (Figure S1).

Taken together, the average number of $\mathrm{H}$-bonded neighbors of a given alcohol molecule as obtained from RDF integration and a H-bond analysis algorithm (hbond), operating on the simulation trajectories, indicate 1-butanol to be more extensively self-associated via $\mathrm{H}$-bonding in its mixtures with $\mathrm{DBE}$ compared to 2-butanol (as well as in the pure liquids), which is generally consistent with the conclusions of previous experimental studies. $^{43}$

In the context of interpreting the different $H^{\mathrm{E}}$ of these mixture series, it is pertinent to compare the extent to which the $\mathrm{H}$ bonded structure of the pure alcohols is broken in the various DBE mixtures. Considering the alcohol-alcohol H-bond number data plotted in Figures 5 and 6, it may be seen that the decrease in the average number of such $\mathrm{H}$-bonds per alcohol molecule follows a steeper decreasing trend (with increasing $\left.x_{\mathrm{DBE}}\right)$ for 2-butanol compared to 1-butanol systems, indicating a greater relative decrease in the number of alcohol-alcohol $\mathrm{H}$ bonds in the former. To facilitate the comparison of the trends, the normalized average number of alcohol-alcohol $\mathrm{H}$-bonds per alcohol molecule for 1-butanol over 2-butanol mixtures, relative to that in the respective pure alcohols, has been calculated and plotted in Figure S2. This normalized number is lower for 2butanol at all studied $x_{\mathrm{DBE}}$, confirming a greater degree of alcohol-alcohol H-bond breakage in 2-butanol + DBE mixtures relative to that in pure 2-butanol compared to 1-butanol mixtures, consistent with the greater $H^{\mathrm{E}}$ of the former at all compositions.

In conclusion, our simulations, which reasonably reproduce the different $H^{\mathrm{E}}$ for 1- and 2-butanol + DBE mixtures, indicate that both a lower degree of alcohol-alcohol H-bond breakage and the participation of a greater fraction of the available alcohol molecules in cross-species $\mathrm{H}$-bonds occur in the 1-butanol mixtures, both of which conceivably account for the lower $H^{\mathrm{E}}$ of these mixtures compared to that of the corresponding 2-butanol mixtures. Evaluation of the relative importance of these contributions requires additional detailed energetic information for the various alcohol-alcohol and alcohol-DBE H-bond types.

We would like to draw attention to a possible limitation of the current computational model. Of the different systems studied here, comparable computer simulation results are available only for the pure liquids, primarily 1-butanol. ${ }^{56,61,63}$ As noted above, while the average number of $\mathrm{H}$-bonded neighbors in pure 1butanol computed from our simulation agrees rather well with that presented by Cerar et al. ${ }^{61}$ (using a different force field and $\mathrm{H}$-bond criteria, the combined effects of which are unknown), the relevant $\mathrm{O}-\mathrm{O} \mathrm{RDF}$ first maximum is notably higher than that of Mesele et al. ${ }^{56}$ Moreover, the 1-butanol nearest-neighbor (H-bonded) coordination number, obtained by $\mathrm{O}-\mathrm{O}$ RDF integration (Figure 5 and Table S6), is also higher than those reported in X-ray and neutron scattering studies. ${ }^{64,65}$ In this context, and considering the too-low simulated $H^{\mathrm{E}}$ for 1-butanol 
mixtures in Figure 2, it appears that the present 1-butanol computational model overestimates alcohol-alcohol $\mathrm{H}$-bonding, both in the pure liquid and its DBE mixtures (also see discussion of $V^{\mathrm{E}}$ below). Since such $\mathrm{H}$-bonding interactions are, within the present computational model, of a purely electrostatic nature, the above limitation points to the $-\mathrm{OH}$ group partial atomic charges ( $\mathrm{O}:-0.71337 e ; \mathrm{H}$ : $+0.43402 e$ ) as possibly being too high (in an absolute sense). As described in the Section 2.4, these partial atomic charges were derived according to an electrostatic potential fitting protocol recommended for GAFF and are, in fact, in very good agreement with those of Caleman et al. (O: $-0.7143 e$; H: $+0.4335 e){ }^{66}$ similarly developed for use with GAFF parameters. Interestingly, Cerar et al. $^{61}$ made use of notably lower $-\mathrm{OH}$ group partial charges (O: $-0.683 e ; \mathrm{H}:+0.418 e$ ) in their comparative MD simulation and $\mathrm{X}$-ray study of pure 1-butanol, also in combination with the AMBER03 force field, which uses the same Lennard-Jones potential parameters as GAFF. Finally, the lower $-\mathrm{OH}$ group partial atomic charges derived for the present 2-butanol model (O: $-0.69586 e ; \mathrm{H}:+0.397918 e$ ) are generally consistent with the Compton scattering experiments and density functional theory (DFT) calculations results of Hakala et al., ${ }^{67}$ which showed that the degree of charge delocalization is greater in the branched iso- and 2-butanol compared to the linear 1-butanol. Notably, this difference in charge distribution is not directly accounted for in the OPLS-AA force field used by Mesele et al., ${ }^{56}$ the only other $\mathrm{MD}$ simulation study to compare the liquid structures of butanol isomers, with all isomers having identical $-\mathrm{OH}$ group partial atomic charges.

4.2. Excess Molar Volumes, Clustering, and Microscopic Heterogeneity. Patil et al. ${ }^{3}$ first reported the excess molar volumes, $V^{\mathrm{E}}$, of all butanol isomer + DBE binary mixtures and interpreted the increasing $V^{\mathrm{E}}$ order 1-butanol (negative) < isobutanol (negative) < 2-butanol (predominantly positive) < tert-butanol (positive) as due to a combination of three contributions.

1. Breaking of alcohol-alcohol H-bonds (structure-breaking), leading to a positive $V^{\mathrm{E}}$.

2. Formation of alcohol-DBE H-bonds, leading to negative $V^{\mathrm{E}}$.

3. Disruption of the orientationally ordered structure of DBE, leading to a positive $V^{\mathrm{E}}$.

The authors ascribed the above $V^{\mathrm{E}}$ trend as resulting from increasing steric hindrance at the alcohol hydroxyl $(-\mathrm{OH})$, correlating the deviation of the alcohol molecular shape from linearity with the reduction of $\mathrm{H}$-bond and van der Waals interactions with the linear DBE. Similar interpretations were presented in subsequent reports of the $V^{\mathrm{E}}$ of these butanol isomer + DBE mixtures by Kammerer and Lichtenthaler ${ }^{4}$ and Bernazzani et al., ${ }^{6}$ focusing mainly on contribution 2 . Interestingly, it appears that the role of contribution 1, i.e., the differences in the degree of breaking up of the characteristic $\mathrm{H}$ bonded structure of the respective pure butanol isomers, has not received the same consideration.

As discussed in the previous section, our simulations indicate that while 1-butanol is more extensively self-associated via $\mathrm{H}$ bonding, a greater degree of breakage of the characteristic pure alcohol H-bonded structure is observed for 2-butanol. Moreover, a greater fraction of non-H-bond saturated 1-butanol molecules, w.r.t. 2-butanol, engage in $\mathrm{H}$-bonds with DBE. These findings are generally consistent (through $V^{\mathrm{E}}$ contributions 1 and 2 described above) with the observed higher $V^{\Xi}$ of 2-butanol
+ DBE mixtures. However, the MD simulations may provide more detailed information on changes in the size distribution of $\mathrm{H}$-bonded alcohol self-associates (clusters) and their topologies, which may, conceivably, also affect the $V^{\mathrm{E}}$ of these mixtures through, e.g., differences in molecular packing efficiency, i.e., contribution 3; in fact, of the systems simulated, only the $\mathrm{H}$ bonded liquid structure of pure 1-butanol has been studied at a comparable level of detail. ${ }^{61,63,68,69}$ With the aim of characterizing the H-bond self-association in the studied mixtures, we developed an algorithm for identifying $\mathrm{H}$-bonded molecular associates, hereafter simply referred to as "clusters", using $\mathrm{H}$ bond criteria $(1,2)$ listed in the previous section. The present $\mathrm{H}$ bond cluster search algorithm is described in detail in the Supporting Information.

The average size (in terms of the number of members) of alcohol H-bonded clusters in the simulated 1- and 2-butanol + DBE mixtures are reported in Table 2. As inferred from the local

Table 2. Average H-Bonded Cluster Sizes in Simulated 1- and 2-Butanol + DBE Mixtures

\begin{tabular}{ccc} 
& \multicolumn{2}{c}{ average cluster size $\left(n_{\mathrm{c}}\right)$} \\
\cline { 2 - 3 }$x_{\mathrm{DBE}}$ & 1-butanol & 2-butanol \\
0.000 & 27.7 & 8.0 \\
0.125 & 16.5 & 6.0 \\
0.250 & 11.1 & 4.7 \\
0.375 & 8.3 & 3.9 \\
0.500 & 6.3 & 3.3 \\
0.625 & 5.0 & 2.7 \\
0.750 & 3.6 & 2.2 \\
0.880 & 2.4 & 1.6 \\
0.940 & 1.7 & 1.3 \\
\hline
\end{tabular}

$\mathrm{H}$-bond analysis presented in the preceding section, the average cluster sizes in 1-butanol and its DBE mixtures are larger than those of the corresponding 2-butanol systems at all compositions studied and decrease with the increasing $x_{\mathrm{DBE}}$ for both mixture series. The average $\mathrm{H}$-bonded cluster size in simulated pure 1-butanol, 27.7, is significantly larger than that reported in previous computer simulation studies $(<4),{ }^{68,69}$ estimated from near-IR measurements $(7.5)^{70}$ and computed using a modified ERAS model (14). ${ }^{71}$ While previous computer simulation studies used different force field models and $\mathrm{H}$-bonded neighbor search criteria, this discrepancy confirms that the present simulations overestimate the extent of 1-butanol $\mathrm{H}$-bonding and self-association, as noted previously. With this possible limitation in mind, as well as the satisfactory reproduction of the essential $V^{\mathrm{E}}$ differences by these simulations (Figure 2), we proceed to consider differences in $\mathrm{H}$-bonded alcohol cluster size distributions in greater detail.

The average count per simulation trajectory frame, or frequency of occurrence, of such H-bonded clusters of a given size (number of members, $n_{\mathrm{c}}$ ) computed for pure 1- and 2butanol and selected DBE mixtures is shown in Figure 7 (leftside panels). In fact, the distribution histograms shown in Figure 7 are mostly only partial, showing the data up to $n_{\mathrm{c}}=10$, whereas for the simulated pure alcohols, the long decaying tails reach sizes $n_{\mathrm{c}}>100$ (very low occurrence); however, as will be shown shortly, we are presently primarily interested in the distribution of smaller alcohol clusters, which account for the majority of selfassociated alcohol molecules in the DBE mixtures (the corresponding full cluster size distributions may be found in the Figure S3, Supporting Information). 

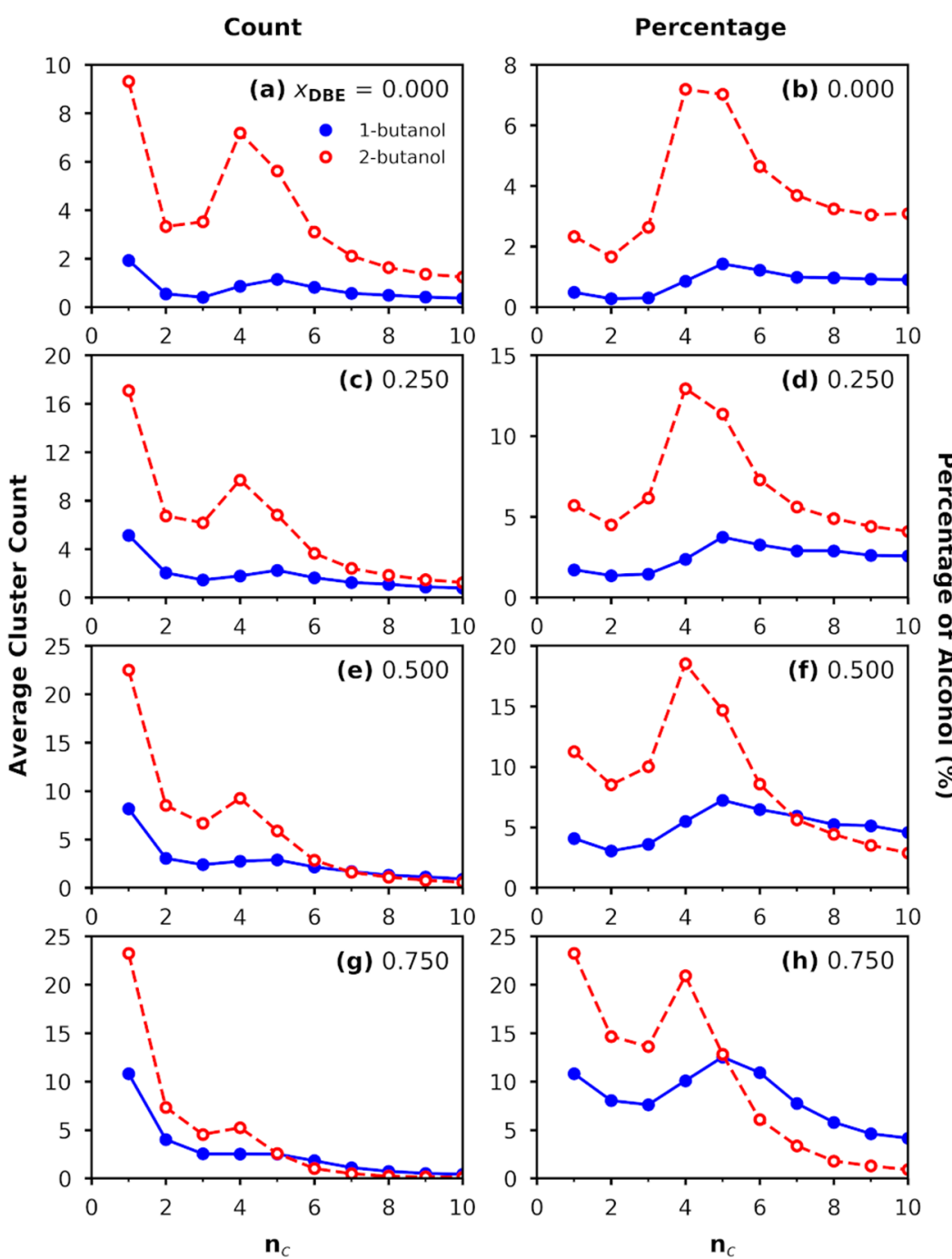

Figure 7. (Left column) Number of H-bonded alcohol clusters of given size $n_{c}$ averaged over the entire production trajectories (1000 configurations) of four representative compositions of 1- and 2-butanol + DBE mixtures, and (right column) the corresponding recast as a percentage of alcohol molecules contributing to $\mathrm{H}$-bonded clusters of a given size.

Considering the distributions obtained for the pure alcohols first, the data set for the simulated 1-butanol may be seen to be consistently lower and flatter, apparently rather featureless, compared to that for 2-butanol. For both 1- and 2-butanol, $n_{\mathrm{c}}=$ 1, i.e., non-H-bonded molecule, has the highest average count, after which significantly lower counts are registered for $\mathrm{H}$ bonded dimers and trimers $\left(n_{c}=2,3\right)$, before increasing for sizes $n_{\mathrm{c}}=4-6$. A similar H-bonded cluster distribution behavior has been previously reported in simulation studies of pure liquid 1hexanol ${ }^{15,16}$ and 1 -octanol $1^{59,72}$ and their aqueous or alkane mixtures and has been ascribed to the increased relative stability of four- to six-membered clusters, specifically cyclic arrangements that allow for an additional alcohol-alcohol $\mathrm{H}$-bond with respect to the noncyclic arrangement; the formation of larger clusters becomes increasingly entropically unfavorable. In fact, such studies often represent the $\mathrm{H}$-bonded cluster size distribution in terms of the average fraction of alcohol molecules occurring in the clusters of given size $n_{c}$; a similar representation of the pure alcohol cluster data is shown in Figure $7 \mathrm{~b}$, and similarly in the right-hand side panels for the remaining mixtures. In Figure $7 b$, it may be seen that non-H-bonded molecules, $n_{\mathrm{c}}=1$, while being the most frequently occurring single cluster size, account for only a small fraction of 1- and 2butanol molecules in the pure liquids. In the case of 1-butanol, a low maximum may be noted at $n_{\mathrm{c}}=5$ (or "pentamer"), while for 2-butanol $n_{\mathrm{c}}=4$ (tetramer, function maximum) and 5 are very prominent. Nevertheless, the majority of molecules in both pure 1 - and 2-butanol occur in larger clusters, $n_{c}>10$, indicating that the properties of these pure liquids are primarily determined by the nature of their extensively $\mathrm{H}$-bonded liquid structures.

Turning to the results for the representative 1- and 2-butanol $+\mathrm{DBE}$ mixtures, $x_{\mathrm{DBE}}=0.25,0.50$, and 0.75 , we note that smaller $\mathrm{H}$-bonded alcohol clusters occur more frequently and account for a greater fraction of alcohol molecules as the larger $\mathrm{H}$ bonded structures are increasingly broken with the increasing DBE content. Interestingly, the $\mathrm{H}$-bonded cluster size preferences observed in Figure 7a,b, i.e., pentamers for 1butanol and tetramers for 2-butanol, appear to persist in the DBE mixtures, with non-self-associated $\left(n_{\mathrm{c}}=1\right)$ alcohol molecules accounting for an increasingly larger alcohol fraction. In fact, at $x_{\mathrm{DBE}}=0.75, \mathrm{H}$-bonded tetramers and pentamers together may be seen in Figure $7 \mathrm{~d}$ to account for $>30 \%$ of 2 butanol molecules in the simulated system. For ease of comparison, the fractions of 1 - and 2-butanol molecules 

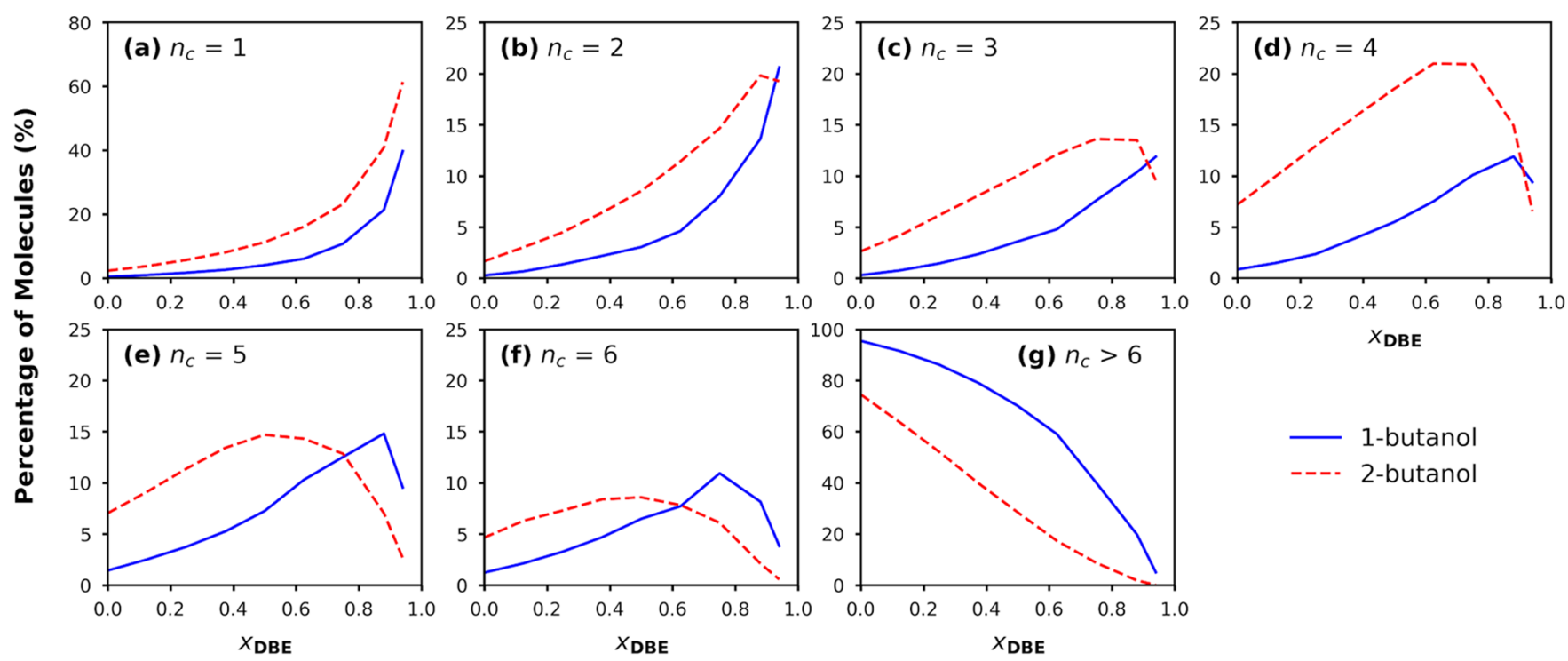

$5(\mathrm{e}), 6(\mathrm{f})$, and $>6(\mathrm{~g})$, plotted as a function of mixture composition, $x_{\mathrm{DBE}}$.

occurring in clusters of size $n_{\mathrm{c}}=1-6$, and $>6$ in all simulated systems are plotted as a function of $x_{\mathrm{DBE}}$ in Figure 8. In these plots, the fractions of 2-butanol molecules participating in smaller clusters, $n_{\mathrm{c}} \leq 4$, are greater than those for 1-butanol over most of the mixture composition range, whereas greater fractions of 1-butanol contribute to large clusters $n_{\mathrm{c}}>6$ at all compositions. The plots for the intermediate cluster sizes, $n_{\mathrm{c}}=$ 4-6, are particularly interesting in that these show clear maxima in the mid- $x_{\mathrm{DBE}}$ composition range, with those of 2-butanol occurring at consistently lower $x_{\mathrm{DBE}}$. The different H-bonded cluster behavior of 1- and 2-butanol in DBE mixtures is visually illustrated by the simulation snapshots in Figure 9. In this figure,
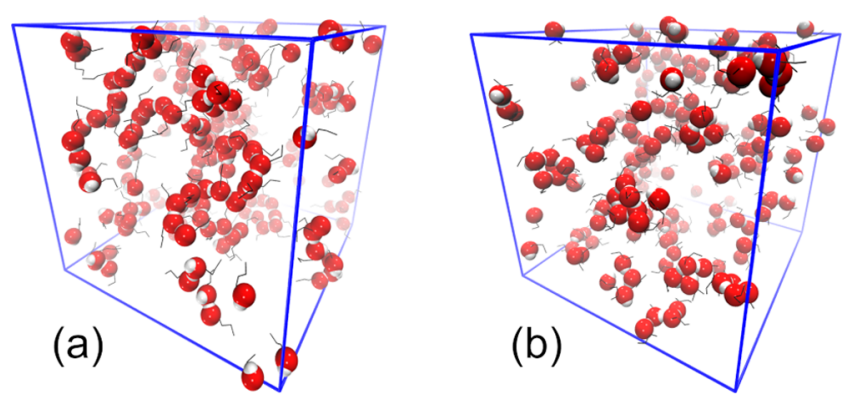

Figure 9. Representative configurations from MD simulations of (a) 1butanol and (b) 2-butanol + DBE mixtures (both with composition $x_{\mathrm{DBE}}=0.5$ ). DBE molecules have been omitted in the interest of clarity, with the butanol $-\mathrm{OH}$ groups shown using a space-filling representation (red and white spheres correspond to $\mathrm{O}$ and $\mathrm{H}$ atoms, respectively). Butanol isomer alkyl groups are shown with $\mathrm{H}$ atoms omitted using a gray line representation.

1-butanol molecules (a) may be seen to engage in extended chain-like $\mathrm{H}$-bonded clusters, whereas 2-butanol tend to form smaller, more compact clusters with a larger number of singlemolecule units $\left(n_{c}=1\right)$, i.e., not H-bonded to other alcohol molecules.

The decrease in the fraction of alcohols in large H-bonded self-associates $\left(n_{\mathrm{c}}>6\right)$ and a concurrent increase in the non-selfassociated $\left(n_{\mathrm{c}}=1\right)$ alcohol fraction with the increasing $x_{\mathrm{DBE}}$ are often proposed for alcohol + alkane binary mixtures, ${ }^{16,71,73}$ as well as the initial increase and a maximum at higher $x_{\mathrm{DBE}}$ of the alcohol fraction participating in small- to intermediate-sized clusters $\left(n_{c}=4-6\right)$. The appearance of the latter type of clusters upon dilution has been explained with the formation of small cyclic H-bonded alcohol clusters. Aida et al., ${ }^{74}$ in their $\mathrm{MD}$ simulation study of volume expansion phenomena in various short-chain alcohol $+\mathrm{CO}_{2}$ binary mixtures, showed a significant fraction of tetrameric $\left(n_{c}=4\right)$ and pentameric $\left(n_{c}=5\right) \mathrm{H}$ bonded alcohol clusters to be simple cycles (or "rings"), the formation of which also feature prominently in their proposed explanation of the phenomenon. Visual inspection of our simulation trajectories has also revealed the presence of such $\mathrm{H}$ bonded alcohol rings, with a higher probability of occurrence in the 2-butanol + DBE mixtures. A representative four-membered H-bonded 2-butanol ring configuration, isolated from the simulation cell configuration in Figure 9, is shown in Figure $10 \mathrm{a}, \mathrm{b}$, where the alkyl groups of the ring members may be seen to adopt alternating up/down relative orientations, likely to minimize steric repulsion and $\mathrm{H}$-bonded ring strain, as had been proposed for cyclic $\mathrm{H}$-bonded tetramers in tert-butanol, its alkane mixtures and vapors. ${ }^{58,75,76}$ A similar 1-butanol configuration shown in Figure 10c,d notably does not exhibit this same alkyl chain orientation (more below).

To more effectively evaluate the importance of simple cyclic $\mathrm{H}$-bonded structures in the self-association of 1- and 2-butanol in their DBE mixtures, an additional algorithm was developed using the previously described $\mathrm{H}$-bond criteria $(1-3)$ to determine the fractions of alcohol molecules present in simple cyclic H-bonded self-associated motifs. The results of this procedure, which initially provides histogram data similar to those in Figure 7, are collected in Figure 11 for selected $\mathrm{H}$ bonded ring sizes $\left(n_{\mathrm{r}}\right)$. The smallest possible $\mathrm{H}$-bonded ring size, $n_{\mathrm{r}}=3$, is notably absent from Figure 11 ; the reason for this omission is that the average fractions of both 1- and 2-butanol molecules participating in such small rings were found to be $<2 \%$ over the entire composition range (Figure S4) likely as a consequence of prohibitive ring strain, suggesting that this particular arrangement likely does not significantly affect the physical properties of the mixtures studied. 

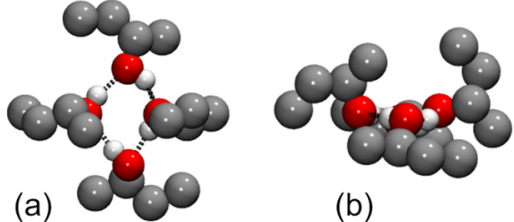

(b)
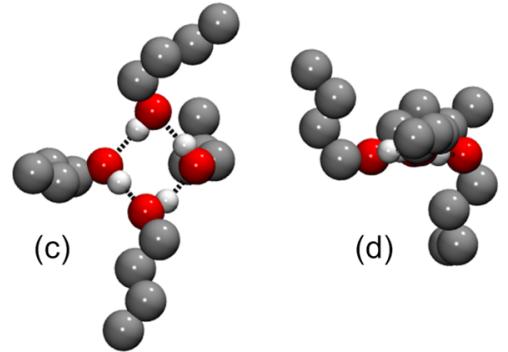

Figure 10. Representative 2-butanol (top row) and 1-butanol (bottom row) four-membered H-bonded ring configuration: $(a, c)$ top views of effective ring planes; (b, d) side views. Aliphatic $\mathrm{H}$ atoms have been omitted in the interest of clarity; $\mathrm{O}$ atoms are in red, $\mathrm{C}$ atoms are in gray, and $\mathrm{H}$ in white. The figures make use of a space-filling ("van der Waals") representation, with default van der Waals radii (VMD) scaled by a factor of 0.5 to better show the H-bonded cluster core. H-bonds are represented as dotted black lines.

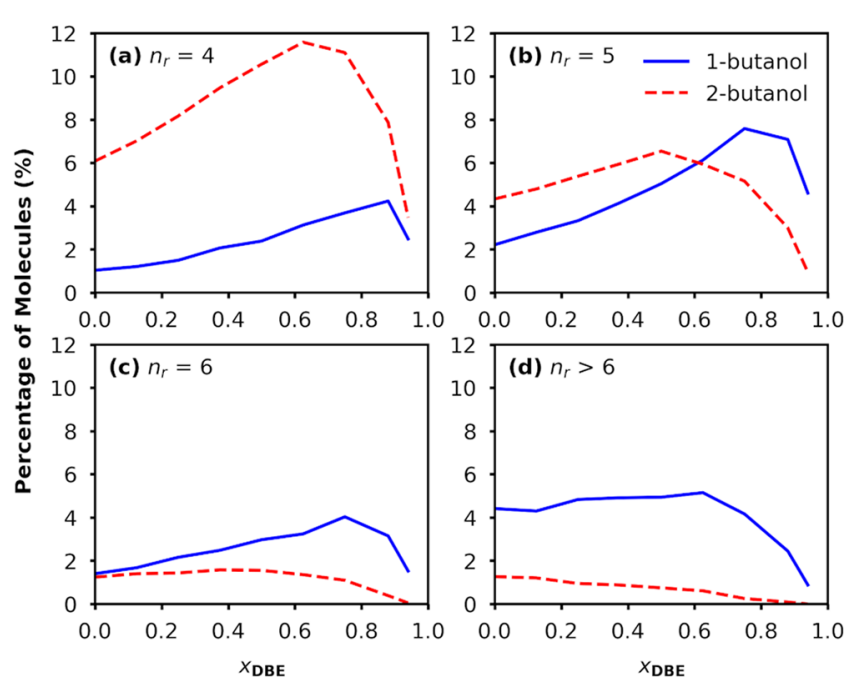

Figure 11. Percentage of 1- (blue solid line) and 2-butanol (red dashed line) molecules in cyclic H-bonded alcohol motifs of size $n_{\mathrm{r}}=4$ (a), 5 (b), 6 (c), and $>6(\mathrm{~d})$, plotted as a function of mixture composition, $x_{\mathrm{DBE}}$

Considering the H-bonded ring sizes $n_{\mathrm{r}}=4$ and 5 in Figure 11, it may be noted that the alcohol fraction variations for both 1and 2-butanol closely resemble those of the corresponding $\mathrm{H}$ bonded cluster sizes, $n_{c}=4$ and 5 , reported in Figure 8, indicating that the significant fractions of these $\mathrm{H}$-bonded rings are, in fact, individual clusters of the corresponding size, as opposed to forming part of larger clusters. Similar findings have been reported by Aida et al. ${ }^{74}$ for 1 - and tert-butanol H-bonded clusters (pentamers and tetramers, respectively) in their simulated binary mixtures with $\mathrm{CO}_{2}$, where, as noted above, the formation of such small cyclic structures were proposed to play an important role in bringing about the lower-thanexpected densities (volumetric expansion) occurring in these mixtures. In fact, comparing the distribution of the 2-butanol fraction in four-membered H-bonded ring motifs $\left(n_{\mathrm{r}}=4\right)$ with the corresponding simulated $V^{£}$ data in Figure 3, similar asymmetric data set shapes may be noted, with maxima in the same mixture composition region $\left(x_{\mathrm{DBE}}=0.625\right)$, suggestive of a possible positive correlation between the presence of such configurations and the average volume of the simulated system.

A preliminary investigation of selected simulation trajectory blocks, spanning tens to hundreds of picoseconds, appears to support the above hypothesis: Figure 12 compares the time-

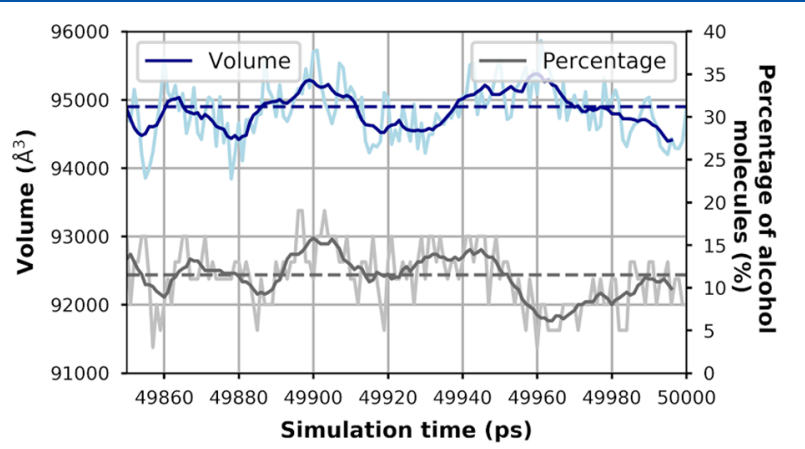

Figure 12. Variation of the simulated system volume (blue, primary $y$ axis) and the number of four-membered $\left(n_{\mathrm{r}}=4\right)$ 2-butanol rings (gray, secondary $y$-axis) over the final $150 \mathrm{ps}$ for a selected 2-butanol + DBE mixture simulation trajectory $\left(x_{\mathrm{DBE}}=0.625\right)$. To facilitate the comparison of variation trends for the two datasets, nine-point running averages have been plotted in bold blue and gray lines for system volume and four-membered ring fraction, respectively. Horizontal dotted black lines show the trajectory average values.

dependent variations in the simulated system volume with those in the number of four-membered $\mathrm{H}$-bonded 2-butanol rings found by our algorithm over the final 150 ps of one of the simulations $\left(x_{\mathrm{DBE}}=0.625\right)$. The main maxima $/$ minima features of these datasets appear to be effectively correlated if somewhat offset. To facilitate comparison of the datasets (which is complicated due to high-frequency fluctuations), running averages (taken over nine datapoints, the original datapoints are separated by 1 ps) are also plotted in Figure 12.

Clearly, a more dedicated computational study is needed to establish the precise nature of this possible correlation, which more than likely involves additional high-volume $\mathrm{H}$-bonded configurations (e.g., five-membered alcohol rings), and may indeed be quite different for 1- and 2-butanol systems. For example, in Figure 12, not all simulation trajectory time blocks with significantly higher-than-average volumes contain high counts of four-membered rings, and it is likely that other configurations contribute to the volume expansion in such instances. Moreover, for 1-butanol + DBE mixtures, which have negative $V^{\Xi}$, there appears to be no apparent correlation between the simulated $V^{\mathrm{E}}$ trend shape and the presence of the H-bonded 1-butanol cluster or ring sizes. The prominent four-membered H-bonded rings of 2-butanol adopt characteristic "up/down" alkyl group configurations shown in Figure 10a,b, which likely serves to relieve the steric repulsion and ring strain produced by these bulky alkyl groups (which is not possible for fivemembered rings, hence their lower occurrence). Such configuration prevents strong H-bonding or dipole interactions of surrounding molecules with the $\mathrm{H}$-bonded core and leads to high-volume structures. ${ }^{74,75}$ Differently, the 1-butanol fourmembered rings, shown in Figure 10c,d, with their more mobile, linear alkyl groups are expected to allow for more favorable interactions with the surrounding molecules. Nevertheless, upon closer inspection of Figures 3 and 11, the increasing prominence 
of four- and, especially, five-membered H-bonded rings $\left(n_{\mathrm{r}}=5\right)$ at $x_{\mathrm{DBE}} \geq 0.75$ do indeed reflect the asymmetry of the simulated $V^{\mathrm{E}}$ trend, which is less-negative in this composition range than expected based on the more highly symmetric experimental data, suggesting a similar positive $V^{\complement}$ contribution by these cyclic structures.

Considering the six-membered and larger $\left(n_{\mathrm{r}}>6\right)$ alcohol $\mathrm{H}$ bonded ring size data in Figure 11c,d, the fraction of 1-butanol molecules involved in such structures may be seen to be greater than that for 2-butanol at all compositions studied. This difference is consistent with the larger average $\mathrm{H}$-bonded alcohol cluster sizes found for the 1-butanol mixtures; however, for these larger $\mathrm{H}$-bonded ring sizes, the trend shapes differ noticeably from those of the corresponding total H-bonded cluster size data $\left(n_{\mathrm{c}}\right)$ in Figure 8, unlike $n_{\mathcal{c}} n_{\mathrm{r}}=4$ and 5 described above. For 1-butanol, larger H-bonded rings $\left(n_{\mathrm{r}}>6\right)$ appear to be rather prominent motifs over a large composition range, with the fraction of 1-butanol molecules contributing to such rings remaining essentially constant up to ca. $x_{\mathrm{DBE}}=0.6$. At any rate, the variation trends of $\mathrm{H}$-bonded ring sizes $n_{\mathrm{r}} \geq 6$ do not appear to correlate with simulated $V^{\mathrm{E}}$ features, with the possible exception of $n_{\mathrm{r}}=6$ in the case of 1-butanol, which shows a shape similar to that of $n_{\mathrm{r}}=5$ (see above); this observation is reasonable in view of the expectation that large cyclic $\mathrm{H}$-bonded structures should not occupy a significantly greater volume compared to a linear or branched $\mathrm{H}$-bonded cluster of the same size (number of members).

Interestingly, a literature survey reveals very few computational reports describing correlations between the specific $\mathrm{H}$ bonded self-associated topologies, e.g., cyclic structures of a given size, and anomalous liquid mixture densities, or $V^{\mathrm{E}}{ }^{74,75}$ On the contrary, the effects of cross-species H-bonding modes on binary liquid mixture excess thermodynamic properties, e.g., leading to negative $V^{\mathrm{E}}$ by interstitial molecular accommodation phenomena, ${ }^{21,48}$ have been studied in detail, notably for aqueous mixtures. In this context, while the computer simulation results presented here largely support the existing interpretations of the different $V^{\mathrm{E}}$ of 1- and 2-butanol + DBE mixtures, namely, as due to more prominent cross-species $\mathrm{H}$ bonding in 1-butanol + DBE mixtures and greater retention of alcohol-alcohol H-bonds upon mixing with $\mathrm{DBE},,^{3,4,6}$ we have shown that changes in the nature of $\mathrm{H}$-bonded alcohol selfassociates, e.g., the increasing fraction of alcohol molecules in cyclic $\mathrm{H}$-bonded clusters with the increasing DBE content of the mixture, may indeed account for an important additional $V^{\mathrm{E}}$ contribution. As noted, more focused computational studies may reveal the precise importance of the presence of such cyclic $\mathrm{H}$-bonded structures and the physical mechanism by which these affect the mixture density, e.g., reduced strength of interactions with the surrounding molecules, poor molecular packing, or inherently high volume of such configurations.

\section{CONCLUSIONS}

1- and 2-Butanol + di-n-butyl ether (DBE) binary liquid mixtures have been studied for the first time by means of extensive MD computer simulations, and the results compared with the newly performed calorimetric and densitometric measurements and with previously reported excess thermodynamic data.

The simulations satisfactorily reproduce the experimentally extensively studied differences in the excess molar enthalpies $\left(H^{\mathrm{E}}\right)$ and volumes $\left(V^{\mathrm{E}}\right)$ of these mixtures and, thus, constitute a reliable model for studying how these properties are related to the differences in the molecular organization.

Extensive structure analysis of the simulated liquid directly demonstrates, for the first time, notable differences in butanol isomer self-association via $\mathrm{H}$-bonding in these mixtures, as well as more subtle differences in butanol-DBE cross-species $\mathrm{H}$ bonding, both of which have been implicated in previous explanations of the excess thermodynamic properties of alcohol-ether binary mixtures. Specifically,

- pure 1-butanol and its DBE mixtures were found to be characterized by larger average $\mathrm{H}$-bonded self-associate sizes and broader size distributions compared to the corresponding 2-butanol mixtures, which prominently feature four- and five-membered $\mathrm{H}$-bonded associates;

- the H-bonded clusters in 1-butanol and its DBE mixtures are more compact, with 1-butanol molecules interacting through shorter $\mathrm{H}$-bonds than those between the 2butanol isomers;

- the higher $H^{\mathrm{E}}$ experimentally observed for 2-butanol$\mathrm{DBE}$ mixtures is explained by the greater relative disruption of $\mathrm{H}$-bonds when $\mathrm{DBE}$ is added to 2-butanol with respect to adding it to 1-butanol, while the number of $\mathrm{H}$-bonds to DBE is essentially the same for the two isomers at any concentration. Taken together, these two observations indicate a larger energetic counterbalance of the reduction in the alcohol self-association by the formation of butanol-DBE H-bonds in 1-butanol mixtures compared to those of 2-butanol;

- a H-bond topological analysis revealed a significant fraction of such small H-bonded 2-butanol clusters to be simple cyclic structures and that the increased prominence of four-membered $\mathrm{H}$-bonded 2-butanol cycles, in particular, appears to show a correlation with the characteristic $V^{\mathrm{E}}$ variation of these mixtures. While a fuller understanding of the effect of small cyclic $\mathrm{H}$-bonded self-associates on the volumetric properties of these mixtures must await a more comprehensive computational study, the present results show that MD simulations can reasonably reproduce the excess thermodynamic properties of these mixtures and provide detailed information on the nature of molecular self-association phenomena.

The results, and the analysis methods employed here, constitute an important step in the study of more complex mixtures, such as those of deep eutectic solvents, which often exhibit interesting $S$-shaped excess molar volumes or enthalpies as a function of mixture composition. ${ }^{77}$

\section{ASSOCIATED CONTENT}

\section{Supporting Information}

The Supporting Information is available free of charge at https://pubs.acs.org/doi/10.1021/acs.jpcb.0c10076.

Experimental and simulated excess thermodynamic data; additional numerical data from RDF and hydrogen bond analyses; description of the hydrogen-bonded cluster search algorithms with additional data; and a description of the block averaging procedure for the estimation of statistical uncertainties for data computed from simulations (PDF) 


\section{AUTHOR INFORMATION}

\section{Corresponding Authors}

Aatto Laaksonen - Centre of Advanced Research in Bionanoconjugates and Biopolymers, Romanian Academy $\bowtie$ Petru Poni (PP) Institute of Macromolecular Chemistry, 00487 Iasi, Romania; Department of Materials and Environmental Chemistry, Division of Physical Chemistry, Arrhenius Laboratory, Stockholm University (SU), 10691 Stockholm, Sweden; State Key Laboratory of Materials-Oriented and Chemical Engineering, Nanjing Tech University (NTU), 210009 Nanjing, China; Department of Engineering Sciences and Mathematics, Division of Energy Science, Luleå University of Technology, SE-97187 Luleå, Sweden; 이이. orid.org/00000001-9783-4535; Email: aatto.laaksonen@mmk.su.se

Silvia Porcedda - Dipartimento di Scienze Chimiche e Geologiche, Università degli Studi di Cagliari, 09042 Cagliari, Italy; Email: porcedda@unica.it

Francesca Mocci - Dipartimento di Scienze Chimiche e Geologiche, Università degli Studi di Cagliari, 09042 Cagliari, Italy; @ orcid.org/0000-0003-1394-9146; Email: fmocci@ unica.it

\section{Authors}

Leon de Villiers Engelbrecht - Dipartimento di Scienze Chimiche e Geologiche, Università degli Studi di Cagliari, 09042 Cagliari, Italy

Riccardo Farris - Dipartimento di Scienze Chimiche e Geologiche, Università degli Studi di Cagliari, 09042 Cagliari, Italy

Tudor Vasiliu - Centre of Advanced Research in Bionanoconjugates and Biopolymers, Romanian Academy $\bowtie$ Petru Poni (PP) Institute of Macromolecular Chemistry, 00487 Iasi, Romania

Monica Demurtas - Dipartimento di Scienze Chimiche e Geologiche, Università degli Studi di Cagliari, 09042 Cagliari, Italy

Alessandra Piras - Dipartimento di Scienze Chimiche e Geologiche, Università degli Studi di Cagliari, 09042 Cagliari, Italy

Flaminia Cesare Marincola - Dipartimento di Scienze Chimiche e Geologiche, Università degli Studi di Cagliari, 09042 Cagliari, Italy; ๑ orcid.org/0000-0002-9695-5957

Complete contact information is available at:

https://pubs.acs.org/10.1021/acs.jpcb.0c10076

\section{Author Contributions}

This manuscript was written through contributions of all authors. All authors have given approval to the final version of the manuscript.

\section{Funding}

This research was funded by Regione Sardegna, grant number RASSR 81788 e codice di Intervento RC CRP 008, by the Ministry of Research and Innovation of Romania (CNCSUEFISCDI, Project Number PN-III-P4-ID-PCCF-2016-0050, within PNCDI III) and by MIUR, Project PRIN2017 "CANDL2" (Grant 2017W75RAE).

\section{Notes}

The authors declare no competing financial interest.

\section{ACKNOWLEDGMENTS}

A.L. acknowledges the Swedish Science Council for financial support. F.M. thanks Prof. Giuseppe Saba and Andrea Ardu for technical support. The computations were enabled by resources provided by the Swedish National Infrastructure for Computing (SNIC) at the PDC center for High Performance Computing , partially funded by the Swedish Research Council through grant agreement no. 2016-07213.

\section{REFERENCES}

(1) Awwad, A. M.; Pethrick, R. A. Ultrasonic Investigations of Mixtures of N-Octane with Isomeric Octanols. J. Chem. Soc., Faraday Trans. 1 1982, 78, 3203-3212.

(2) Pathak, G.; Pradhan, S. Enthalpies of Mixing of Di-n-Butyl Ether with Isomeric Butanols: Enthalpies of Hydrogen-Bonded Complexes. Proc. Indian Acad. Sci., Chem. Sci. 1988, 100, 519-523.

(3) Patil, K. R.; Pathak, G.; Pradhan, S. D. Excess Volumes of Isomeric Butanols with Di-n-Butyl Ether. Proc. Indian Acad. Sci., Chem. Sci. 1989, 101, 443-447.

(4) Kammerer, K.; Lichtenthaler, R. N. Excess Properties of Binary Alkanol-Ether Mixtures and the Application of the ERAS Model. Thermochim. Acta 1998, 310, 61-67.

(5) Rezanova, E. N.; Kammerer, K.; Lichtenthaler, R. N. Excess Properties of Binary Alkanol + Diisopropyl Ether (DIPE) or + Dibutyl Ether (DBE) Mixtures and the Application of the Extended Real Associated Solution Model. J. Chem. Eng. Data 1999, 44, 1235-1239.

(6) Bernazzani, L.; Carosi, M. R.; Duce, C.; Gianni, P.; Mollica, V. Volumetric Properties of Binary Mixtures of Isomeric Butanols and C8 Solvents at 298.15 K. J. Solution Chem. 2006, 35, 1567-1585.

(7) Alaoui, F. E. M.; Montero, E. A.; Bazile, J.-P.; Aguilar, F.; Boned, C. Liquid Density of Biofuel Mixtures: (Dibutyl Ether+1-Butanol) System at Pressures up to $140 \mathrm{MPa}$ and Temperatures from (293.15 to 393.15)K. J. Chem. Thermodyn. 2011, 43, 1768-1774.

(8) Alaoui, F.; Montero, E.; Bazile, J. P.; Comuñas, M. J. P.; Galliero, G.; Boned, C. Liquid Density of 1-Butanol at Pressures up to $140 \mathrm{MPa}$ and from 293.15 K to 403.15 K. Fluid Phase Equilib. 2011, 301, 131136.

(9) Alaoui, F. E. M.; Montero, E. A.; Bazile, J. P.; Aguilar, F.; Boned, C. Liquid Density of Biofuel Additives: 1-Butoxybutane at Pressures up to $140 \mathrm{MPa}$ and from (293.15 to 393.15) K. J. Chem. Eng. Data 2011, 56, 595-600.

(10) Dakkach, M.; Aguilar, F.; Alaoui, F. E. M.; Montero, E. A. Liquid Density of Oxygenated Additives to Biofuels: 2-Butanol at Pressures up to $140 \mathrm{MPa}$ and Temperatures from (293.15 to 393.27) K. J. Chem. Thermodyn. 2015, 89, 278-285.

(11) Directive 2009/28/EC of the European Parliament and of the Council of 23 April 2009 on the Promotion of the Use of Energy from Renewable Sources and Amending and Subsequently Repealing Directives 2001/77/EC and 2003/30/EC, 2015.

(12) González, J. A.; Carmona, F. J.; Riesco, N.; De La Fuente, I. G.; Cobos, J. C. Thermodynamics of Mixtures Containing Ethers. Part I. DISQUAC Characterization of Systems of MTBE, TAME or ETBE with n-Alkanes, Cyclohexane, Benzene, Alkan-1-ols or Alkan-2-ols. Comparison with Dortmund UNIFAC Results. Phys. Chem. Chem. Phys. 2000, 2, 2587-2597.

(13) Murdoch, K. M.; Ferris, T. D.; Wright, J. C.; Farrar, T. C. Infrared Spectroscopy of Ethanol Clusters in Ethanol-Hexane Binary Solutions. J. Chem. Phys. 2002, 116, 5717-5724.

(14) Balanay, M. P.; Kim, D. H.; Fan, H. Revisiting the Formation of Cyclic Clusters in Liquid Ethanol. J. Chem. Phys. 2016, 144, No. 154302.

(15) Stubbs, J. M.; Chen, B.; Potoff, J. J.; Siepmann, J. I. Monte Carlo Calculations for the Phase Equilibria of Alkanes, Alcohols, Water, and Their Mixtures. Fluid Phase Equilib. 2001, 183-184, 301-309.

(16) Stubbs, J. M.; Siepmann, J. I. Elucidating the Vibrational Spectra of Hydrogen-Bonded Aggregates in Solution: Electronic Structure Calculations with Implicit Solvent and First-Principles Molecular Dynamics Simulations with Explicit Solvent for 1-Hexanol in n-Hexane. J. Am. Chem. Soc. 2005, 127, 4722-4729. 
(17) Gómez-Álvarez, P.; Romaní, L.; González-Salgado, D. Association Effects in the \{Methanol + Inert Solvent $\}$ System via Monte Carlo Simulations. I. Structure. J. Chem. Phys. 2013, 138, No. 204505.

(18) Fouad, W. A.; Wang, L.; Haghmoradi, A.; Gupta, S. K.; Chapman, W. G. Understanding the Thermodynamics of Hydrogen Bonding in Alcohol-Containing Mixtures: Self Association. J. Phys. Chem. B 2015, 119, 14086-14101.

(19) Požar, M.; Lovrinčević, B.; Zoranić, L.; Primorać, T.; Sokolić, F.; Perera, A. Micro-Heterogeneity: Versus Clustering in Binary Mixtures of Ethanol with Water or Alkanes. Phys. Chem. Chem. Phys. 2016, 18, 23971-23979.

(20) Essafri, I.; Ghoufi, A. Microstructure of Nonideal Methanol Binary Liquid Mixtures. Phys. Rev. E 2019, 99, 1-12.

(21) Kgagodi, O. W.; Mbaiwa, F. Molecular Dynamics Study of 2,2'Difurylmethane and $n$-Propanol Binary Mixture. J. Mol. Liq. 2017, 227, 366-372.

(22) Riddick, J. A.; Bunger, W. B.; Sakano, T. K. Organic Solvents: Physical Properties and Methods of Purification, 4th ed.; WileyInterscience: New York, NY, 1986.

(23) Wieser, M. E. Atomic Weights of the Elements 2005 (IUPAC Technical Report). Pure Appl. Chem. 2006, 78, 2051-2066.

(24) Stokes, R. H.; Marsh, K. N.; Tomlins, R. P. An Isothermal Displacement Calorimeter for Endothermic Enthalpies of Mixing. 1969, 1,211-221. J. Chem. Thermodyn. 1969, 1, 211-221.

(25) Marsh, K.; Richards, A. Excess Volumes for Ethanol + Water Mixtures at $10-\mathrm{K}$ Intervals from 278.15 to $338.15 \mathrm{~K}$. Aust. J. Chem. 1980, 33, 2121-2132.

(26) Case, D. A.; Ben-Shalom, I. Y.; Brozell, S. R.; Cerutti, D. S.; Cheatham, T. E. I.; Cruzeiro, V. W. D.; Darden, T. A.; Duke, R. E.; Ghoreishi, D.; Gilson, M. K.; Gohlke, H.; Goetz, A. W.; Greene, D.; Harris, R.; Homeyer, N.; Huang, Y.; Izadi, S.; Kovalenko, A.; Kurtzman, T.; Lee, T. S.; LeGrand, S.; Li, P.; Lin, C.; Liu, J.; Luchko, T.; Luo, R.; Mermelstein, D. J.; Merz, K. M.; Miao, Y.; Monard, G.; Nguyen, C.; Nguyen, H.; Omelyan, I.; Onufriev, A.; Pan, F.; Qi, R.; Roe, D. R.; Roitberg, A.; Sagui, C.; Schott-Verdugo, S.; Shen, J.; Simmerling, C. L.; Smith, J.; Ferrer, R. S.; Swails, J.; Walker, R. C.; Wang, J.; Wei, H.; Wolf, R. M.; Wu, X.; Xiao, L.; York, D. M.; Kollman, P. A. AMBER 2018; University of California: San Francisco, 2018.

(27) Le Grand, S.; Götz, A. W.; Walker, R. C. SPFP: Speed without Compromise - A Mixed Precision Model for GPU Accelerated Molecular Dynamics Simulations. Comput. Phys. Commun. 2013, 184, 374-380.

(28) Wang, J.; Wolf, R. M.; Caldwell, J. W.; Kollman, P. A.; Case, D. A. Development and Testing of a General Amber Force Field. J. Comput. Chem. 2004, 25, 1157-1174.

(29) Frisch, M. J.; Trucks, G. W.; Schlegel, H. B.; Scuseria, G. E.; Robb, M. A.; Cheeseman, J. R.; Scalmani, G.; Barone, V.; Mennucci, B.; Petersson, G. A.; Nakatsuji, H.; Caricato, M.; Li, X.; Hratchian, H. P.; Izmaylov, A. F.; Bloino, J.; Zheng, G.; Sonnenberg, J. L.; Hada, M.; Ehara, M.; Toyota, K.; Fukuda, R.; Hasegawa, J.; Ishida, M.; Nakajima, T.; Honda, Y.; Kitao, O.; Nakai, H.; Vreven, T.; Montgomery, J. A.; Peralta, J. E.; Ogliaro, F.; Bearpark, M.; Heyd, J. J.; Brothers, E.; Kudin, K. N.; Staroverov, V. N.; Kobayashi, R.; Normand, J.; Raghavachari, K.; Rendell, A.; Burant, J. C.; Iyengar, S. S.; Tomasi, J.; Cossi, M.; Rega, N.; Millam, J. M.; Klene, M.; Knox, J. E.; Cross, J. B.; Bakken, V.; Adamo, C.; Jaramillo, J.; Gomperts, R.; Stratmann, R. E.; Yazyev, O.; Austin, A. J.; Cammi, R.; Pomelli, C.; Ochterski, J. W.; Martin, R. L.; Morokuma, K.; Zakrzewski, V. G.; Voth, G. A.; Salvador, P.; Dannenberg, J. J.; Dapprich, S.; Daniels, A. D.; Farkas, Ö.; Foresman, J. B.; Ortiz, J. V.; Cioslowski, J.; Fox, D. J. Gaussian 09, revision C.01; Gaussian Inc., 2009.

(30) Berendsen, H. J. C.; Postma, J. P. M.; van Gunsteren, W. F.; DiNola, A.; Haak, J. R. Molecular Dynamics with Coupling to an External Bath. J. Chem. Phys. 1984, 81, 3684-3690.

(31) Harvey, S. C.; Tan, R. K.-Z.; Cheatham, T. E., III The Flying Ice Cube: Velocity Rescaling in Molecular Dynamics Leads to Violation of Energy Equipartition. J. Comput. Chem. 1998, 19, 726-740.

(32) Martínez, L.; Andrade, R.; Birgin, E. G.; Martínez, J. M. PACKMOL: A Package for Building Initial Configurations for
Molecular Dynamics Simulations. J. Comput. Chem. 2009, 30, 21572164.

(33) Ryckaert, J. P.; Ciccoti, G.; Berendsen, H. J. C. Numerical Integration of the Cartesian Equation of a System with Constraints: Molecular Dynamics of $n$-Alkanes. J. Comput. Phys. 1977, 23, 327-341.

(34) Flyvbjerg, H.; Petersen, H. G. Error Estimates on Averages of Correlated Data. J. Chem. Phys. 1989, 91, 461.

(35) Humphrey, W.; Dalke, A.; Schulten, K. VMD - Visual Molecular Dynamics. J. Mol. Graphics 1996, 14, 33-38.

(36) Bernazzani, L.; Ceccanti, N.; Conti, G.; Gianni, P.; Mollica, V.; Tinè, M. R.; Lepori, L.; Matteoli, E.; Spanedda, A. Volumetric Properties of (an Organic Compound + Di- $n$-Butyl Ether) at $\mathrm{T}=$ 298.15 Ka. J. Chem. Thermodyn. 2001, 33, 629-641.

(37) Jorgensen, W. L.; Maxwell, D. S.; Tirado-Rives, J. Development and Testing of the OPLS All-Atom Force Field on Conformational Energetics and Properties of Organic Liquids. J. Am. Chem. Soc. 1996, $118,11225-11236$.

(38) Dai, J.; Li, X.; Zhao, L.; Sun, H. Enthalpies of Mixing Predicted Using Molecular Dynamics Simulations and OPLS Force Field. Fluid Phase Equilib. 2010, 289, 156-165.

(39) Miroshnichenko, S.; Vrabec, J. Excess Properties of Non-Ideal Binary Mixtures Containing Water, Methanol and Ethanol by Molecular Simulation. J. Mol. Liq. 2015, 212, 90-95.

(40) Murthy, A. S. N.; Rao, C. N. R. Spectroscopic Studies of the Hydrogen Bond. Appl. Spectrosc. Rev. 1968, 2, 69-191.

(41) Valén, A.; López, M. C.; Urieta, J. S.; Royo, F. M.; Lafuente, C. Thermodynamic Study of Mixtures Containing Oxygenated Compounds. J. Mol. Liq. 2002, 95, 157-165.

(42) Giner, B.; Artigas, H.; Carrión, A.; Lafuente, C.; Royo, F. M. Excess Thermodynamic Properties of Isomeric Butanols with 2Methyl-Tetrahydrofuran. J. Mol. Liq. 2003, 108, 303-311.

(43) Rytting, J. H.; Anderson, B. D.; Higuchi, T. Vapor Pressure Studies of the Self-Association of Alcohols in Isooctane. 2. The Effect of Chain Branching. J. Phys. Chem. A. 1978, 82, 2240-2245.

(44) Chaudhari, S. K.; Katti, S. S. Excess Volumes of (an Isomer of Butanol + n-Octane) at $298.15 \mathrm{~K}$, Measured with a ContinuousDilution Dilatometer. J. Chem. Thermodyn. 1985, 17, 101-104.

(45) Kusalik, P. G.; Lyubartsev, A. P.; Bergman, D. L.; Laaksonen, A. Computer Simulation Study of tert-Butyl Alcohol. 1. Structure in the Pure Liquid. J. Phys. Chem. B 2000, 104, 9526-9532.

(46) Kusalik, P. G.; Lyubartsev, A. P.; Bergman, D. L.; Laaksonen, A. Computer Simulation Study of tert-Butyl Alcohol. 2. Structure in Aqueous Solution. J. Phys. Chem. B 2000, 104, 9533-9539.

(47) Fornili, A.; Civera, M.; Sironi, M.; Fornili, S. L. Molecular Dynamics Simulation of Aqueous Solutions of Trimethylamine-NOxide and tert-Butyl Alcohol. Phys. Chem. Chem. Phys. 2003, 5, 49054910.

(48) Usula, M.; Mocci, F.; Marincola, F. C. C.; Porcedda, S.; Gontrani, L.; Caminiti, R. The Structural Organization of N-Methyl-2Pyrrolidone + Water Mixtures: A Densitometry, X-ray Diffraction, and Molecular Dynamics Study. J. Chem. Phys. 2014, 140, No. 124503.

(49) Usula, M.; Porcedda, S.; Mocci, F.; Gontrani, L.; Caminiti, R.; Marincola, F. C. NMR, Calorimetry, and Computational Studies of Aqueous Solutions of N-Methyl-2-Pyrrolidone. J. Phys. Chem. B 2014, 118, 10493-10502.

(50) Vishnyakov, A.; Laaksonen, A. Carbohydrates Exhibit a Distinct Preferential Solvation Pattern in Binary Aqueous Solvent Mixtures. Angew. Chem., Int. Ed. 2000, 39, 1998-2000.

(51) Engelbrecht, L.; Mocci, F.; Laaksonen, A.; Koch, K. R. K. R. ${ }^{195} \mathrm{Pt}$ NMR and Molecular Dynamics Simulation Study of the Solvation of $\left[\mathrm{PtCl}_{6}\right]^{2-}$ in Water-Methanol and Water-Dimethoxyethane Binary Mixtures. Inorg. Chem. 2018, 57, 12025-12037.

(52) Wang, Y. L.; Li, B.; Sarman, S.; Mocci, F.; Lu, Z. Y.; Yuan, J.; Laaksonen, A.; Fayer, M. D. Microstructural and Dynamical Heterogeneities in Ionic Liquids. Chem. Rev. 2020, 120, 5798-5877.

(53) Laaksonen, A.; Lyubartsev, A.; Mocci, F. M.DynaMix Studies of Solvation, Solubility and Permeability. In Molecular Dynamics-Studies of Synthetic and Biological Macromolecules; Wang, P. L., Ed.; Intech, 2012; pp 85-103. 
(54) Mariani, A.; Caminiti, R.; Ramondo, F.; Salvitti, G.; Mocci, F.; Gontrani, L. Inhomogeneity in Ethylammonium Nitrate-Acetonitrile Binary Mixtures: The Highest "Low q Excess" Reported to Date. J. Phys. Chem. Lett. 2017, 8, 3512-3522.

(55) Head-Gordon, T.; Hura, G. Water Structure from Scattering Experiments and Simulation. Chem. Rev. 2002, 102, 2651-2670.

(56) Mesele, O. O.; Vartia, A. A.; Laage, D.; Thompson, W. H. Reorientation of Isomeric Butanols: The Multiple Effects of Steric Bulk Arrangement on Hydrogen-Bond Dynamics. J. Phys. Chem. B 2016, 120, 1546-1559.

(57) Luzar, A.; Chandler, D. Effect of Environment on Hydrogen Bond Dynamics in Liquid Water. Phys. Rev. Lett. 1996, 76, 928-931.

(58) Andanson, J. M.; Soetens, J. C.; Tassaing, T.; Besnard, M. Hydrogen Bonding in Supercritical tert-Butanol Assessed by Vibrational Spectroscopies and Molecular-Dynamics Simulations. J. Chem. Phys. 2005, 122, No. 174512.

(59) DeBolt, S. E.; Kollman, P. A. Investigation of Structure, Dynamics, and Solvation in 1-Octanol and Its Water-Saturated Solution: Molecular Dynamics and Free-Energy Perturbation Studies. J. Am. Chem. Soc. 1995, 117, 5316-5340.

(60) Roe, D. R.; Cheatham, T. E. PTRAJ and CPPTRAJ: Software for Processing and Analysis of Molecular Dynamics Trajectory Data. J. Chem. Theory Comput. 2013, 9, 3084-3095.

(61) Cerar, J.; Lajovic, A.; Jamnik, A.; Tomšič, M. Performance of Various Models in Structural Characterization of $n$-Butanol: Molecular Dynamics and X-Ray Scattering Studies. J. Mol. Liq. 2017, 229, 346357.

(62) Van Der Spoel, D.; Lindahl, E.; Hess, B.; Groenhof, G.; Mark, A. E.; Berendsen, H. J. C. GROMACS: Fast, Flexible, and Free. J. Comput. Chem. 2005, 26, 1701-1718.

(63) Lehtola, J.; Hakala, M.; Hämäläinen, K. Structure of Liquid Linear Alcohols. J. Phys. Chem. B 2010, 114, 6426-6436.

(64) Shmyt'Ko, I. M.; Jiménez-Riobóo, R. J.; Hassaine, M.; Ramos, M. A. Structural and Thermodynamic Studies of $n$-Butanol. J. Phys.: Condens. Matter 2010, 22, No. 195102.

(65) Cuello, G. J.; Cristiglio, V.; González, M. A.; Cabrillo, C. Structure Factor of Liquid $n$-Butanol at Room Temperature. J. Phys. Conf. Ser. 2014, 549, No. 012015.

(66) Caleman, C.; Van Maaren, P. J.; Hong, M.; Hub, J. S.; Costa, L. T.; Van Der Spoel, D. Force Field Benchmark of Organic Liquids: Density, Enthalpy of Vaporization, Heat Capacities, Surface Tension, Isothermal Compressibility, Volumetric Expansion Coefficient, and Dielectric Constant. J. Chem. Theory Comput. 2012, 8, 61-74.

(67) Hakala, M.; Nygård, K.; Vaara, J.; Itou, M.; Sakurai, Y.; Hämäläinen, K. Charge Localization in Alcohol Isomers Studied by Compton Scattering. J. Chem. Phys. 2009, 130, No. 034506.

(68) Sillrén, P.; Bielecki, J.; Mattsson, J.; Börjesson, L.; Matic, A. A Statistical Model of Hydrogen Bond Networks in Liquid Alcohols. J. Chem. Phys. 2012, 136, No. 094514.

(69) Janeček, J.; Paricaud, P. Size Distribution of Associated Clusters in Liquid Alcohols: Interpretation of Simulation Results in the Frame of SAFT Approach. J. Chem. Phys. 2013, 139, No. 174502.

(70) Iwahashi, M.; Suzuki, M.; Katayama, N.; Matsuzawa, H.; Czarnecki, M. A.; Ozaki, Y.; Wakisaka, A. Molecular Self-Assembling of Butan-1-ol, Butan-2-ol, and 2-Methylpropan-2-Ol in Carbon Tetrachloride Solutions as Observed by near-Infrared Spectroscopic Measurements. Appl. Spectrosc. 2000, 54, 268-276.

(71) Wandschneider, D.; Michalik, M.; Heintz, A. Spectroscopic and Thermodynamic Studies of Liquid $n$-Butanol $+n$-Hexane and + Cyclohexane Mixtures Based on Quantum Mechanical Ab Initio Calculations of n-Butanol Clusters. J. Mol. Liq. 2006, 125, 2-13.

(72) MacCallum, J. L.; Tieleman, D. P. Structures of Neat and Hydrated 1-Octanol from Computer Simulations. J. Am. Chem. Soc. 2002, 124, 15085-15093.

(73) Henkel, S.; Misuraca, M. C.; Troselj, P.; Davidson, J.; Hunter, C. A. Polarisation Effects on the Solvation Properties of Alcohols. Chem. Sci. 2018, 9, 88-99.
(74) Aida, T.; Aizawa, T.; Kanakubo, M.; Nanjo, H. Relation between Volume Expansion and Hydrogen Bond Networks for $\mathrm{CO}_{2}$-Alcohol Mixtures at $40^{\circ} \mathrm{C}$. J. Phys. Chem. B 2010, 114, 13628-13636.

(75) Sassi, P.; Palombo, F.; Cataliotti, R. S.; Paolantoni, M.; Morresi, A. Distributions of H-Bonding Aggregates in tert-Butyl Alcohol: The Pure Liquid and Its Alkane Mixtures. J. Phys. Chem. A 2007, 111, 60206027.

(76) Fanourgakis, G. S.; Shi, Y. J.; Consta, S.; Lipson, R. H. A Spectroscopic and Computer Simulation Study of Butanol Vapors. J. Chem. Phys. 2003, 119, 6597-6608.

(77) Ma, C.; Laaksonen, A.; Liu, C.; Lu, X.; Ji, X. The Peculiar Effect of Water on Ionic Liquids and Deep Eutectic Solvents. Chem. Soc. Rev. 2018, 47, 8685-8720. 